\title{
Republic of Lithuania: Statistical Appendix
}

This Statistical Appendix paper for the Republic of Lithuania was prepared by a staff team of the International Monetary Fund as background documentation for the periodic consultation with the member country. It is based on the information available at the time it was completed on July 28, 2003. The views expressed in this document are those of the staff team and do not necessarily reflect the views of the government of the Republic of Lithuania or the Executive Board of the IMF.

The policy of publication of staff reports and other documents by the IMF allows for the deletion of market-sensitive information.

To assist the IMF in evaluating the publication policy, reader comments are invited and may be sent by e-mail to publicationpolicy@imf.org.

Copies of this report are available to the public from

International Monetary Fund - Publication Services

$70019^{\text {th }}$ Street, N.W. - Washington, D.C. 20431

Telephone: (202) 623-7430 • Telefax: (202) 623-7201

E-mail: publications@imf.org • Internet: http://www.imf.org

Price: $\$ 15.00$ a copy

\section{International Monetary Fund \\ Washington, D.C.}




\title{
INTERNATIONAL MONETARY FUND
}

\author{
REPUBLIC OF LITHUANIA \\ Statistical Appendix \\ Prepared by Patricia Alonso-Gamo, Stefania Fabrizio, \\ Tobias Rasmussen, Ramdane Abdoun, Haiyan Shi (all EU2), and \\ Ioannis Halikias (PDR) \\ Approved by European II Department
}

July 28,2003

Contents

Page

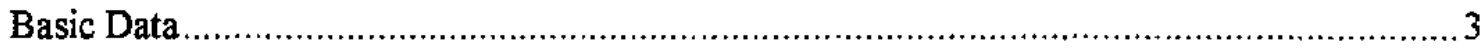

Statistical Appendix Tables

1. Key Economic and Financial Indicators, 1998-2002 ........................................ 4

2. Gross Value Added by Sector at Current Prices, 1998-2002 .................................

3. Gross Value Added by Sector at 2000 Constant Prices, 1998-2002 ......................6

4. Gross Domestic Product by Expenditure at Current Prices, 1998-2002 ................... 7

5. Gross Domestic Product by Expenditure at 2000 Constant Prices, 1998-2002 _........ 8

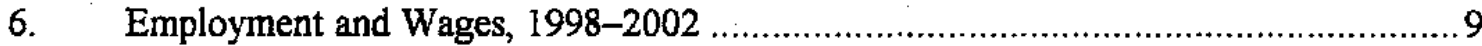

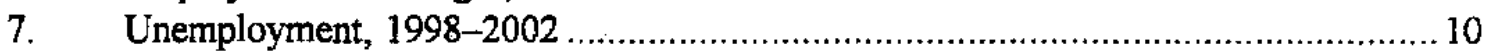

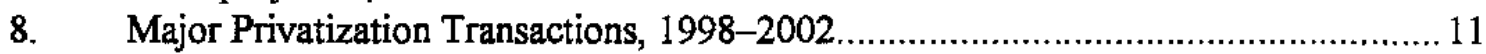

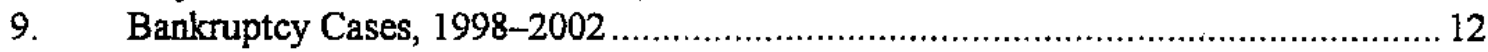

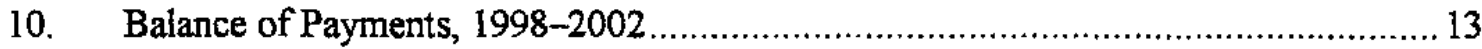

11. Merchandise Trade by Commodity Group, 1998-2002 .................................... 14

12. Merchandise Trade by Country and Country Group, 1998-2002 …........................ 15

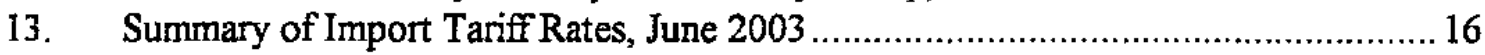

14. Stock of Foreign Direct Investment by Sector, 1998-2002 .............................. 17

15. Stock of Foreign Direct Investment by Source Country, 1998-2002 .................... 18

16. Foreign Direct Investment Inflows, 1998-2002 ........................................... 19

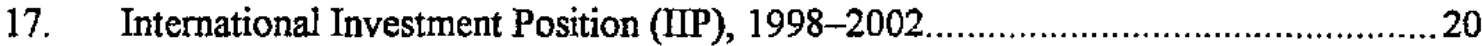

18. Indicators of External and Financial Vulnerability, 1999-2003 ...........................21

19. Summary of Consolidated General Government Operations, $1998-2002$................22 
20. Summary of Consolidated General Government Operations, 1998-2002 ................ 23

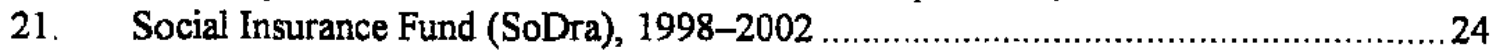

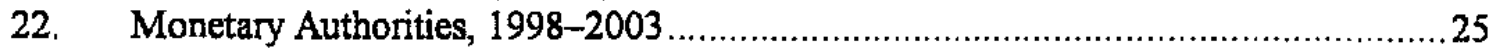

23. Deposit Money Banks, 1998-2003 ............................................................ 26

24. Summary Monetary Accounts, 1998-2003 …............................................ 27

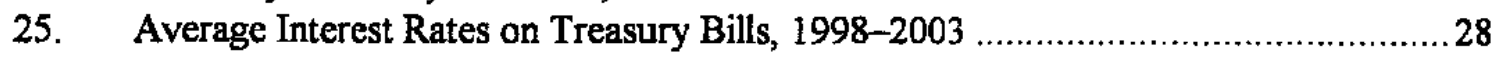

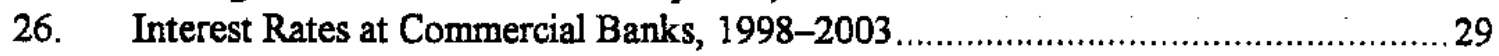

27. Indicators of Financial Sector Vulnerability, 1998-2003 ................................... 30

Appendix

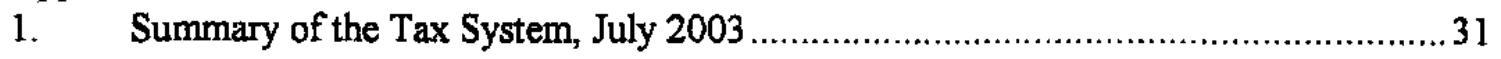


Lithuania: Basic Data

$\begin{array}{lr}\text { Social and demographic indicators (latest available dat2) } \\ \text { Aruz (uq. km) } & 65,300 \\ \text { Population density (inhabitants per sq. km.) } & 53.0 \\ \text { Popuintion (thousanda) } & 3,475.6 \\ \text { Life expectancy at birth 1/ } & 71.7 \\ \text { Men } & 65.9 \\ \text { Wemen } & 77.4 \\ \text { Infant montality rate (per 1,000 birth) 1/ } & 8.0 \\ \text { Hompital beds (per 1,000 inhabitants) 1/ } & 9.2 \\ \text { Phyricians (per 1,000 inhzbitants) 1/ } & 4.0\end{array}$

\begin{tabular}{rrrrr}
1998 & 1999 & 2000 & 2001 & 2002 \\
\hline
\end{tabular}

National accounts

Domeatic dermand

Conumption

Grous inveatment

External balance of gooda and aervices

(in percent of GDP)

GDP

Real GDP

GDP (in millions of litai)

GDP per capita (in litai)

Contumer prices (end-period)

Resl wage 2

Laber market

TotsI miploymont (thomands) 3/

Of which: in itsto wetor

Unenoloyment rate (in perceni) 4

Balance of payments

Trade briarece

Currant account balance

Morry and credit

Broad moary

Credit to private aector

Enterent rate (in percent per ansum) s/

General government finances 6

Revenue and grants

Exponditure and net lending

Finencial balance 7

Ficent belance 7

$\begin{array}{rrrrr}111.7 & 110.3 & 106.5 & 105.5 & 105.7 \\ 85.4 & 87.2 & 86.3 & 84.5 & 83.0 \\ 26.3 & 23.1 & 20.2 & 21.0 & 22.6 \\ -11.7 & -10.3 & -6.5 & -5.5 & -5.7 \\ 100 & 100 & 100 & 100 & 100\end{array}$

(Percentage change from previaus period)

$\begin{array}{rrrrr}7.3 & -1.8 & 4.0 & 6.5 & 6.7 \\ 43,555 & 42,608 & 44,698 & 47,498 & 50,679 \\ 12,227 & 12,048 & 12,727 & 13,621 & 14,581 \\ 2.4 & 0.3 & 1.5 & 2.0 & -1.0 \\ 13.7 & 5.4 & -2.6 & -0.2 & 5.0 \\ & & & & \\ 1,656 & 1,648 & 1,586 & 1,352 & 1,406 \\ 528 & 512 & 486 & 453 & 423 \\ 6.4 & 8.4 & 11.5 & 12.5 & 11.3\end{array}$

(In milliens of U.S. dollers)

$$
\begin{array}{rrrrr}
-1,518 & -1,405 & -1,104 & -1,108 & -1,337 \\
-1,298 & -1,194 & -675 & -574 & -734 \\
\multicolumn{4}{c}{} \\
\text { (Percentage change from previous period) }
\end{array}
$$

$\begin{array}{rrrrr}14.5 & 7.7 & 16.5 & 21.4 & 16.9 \\ 16.9 & 13.8 & -1.2 & 15.4 & 30.4 \\ 12.0 & 13.0 & 11.9 & 9.3 & 6.6\end{array}$

(In percent of GDP)

\begin{tabular}{lllll}
32.7 & 32.1 & 30.7 & 30.1 & 30.5 \\
38.1 & 40.3 & 33.6 & 31.7 & 31.4 \\
-4.4 & -6.2 & -2.1 & -1.8 & -1.4 \\
-5.9 & -8.5 & -2.8 & -2.0 & -1.2 \\
\hline
\end{tabular}

Sources: Lithmanian uuthorities; and Fund ataff estimates.

1/ Data as of 2001 .

2 Averge wage deflated by corsumer price inder.

3/ Figures for 2001 and 2002 reflect a downward revision of the extimated population

4 Criculnted on the basis of regintered unemployment; period averge.

5/ Average anonl interest rate on loun in domeatic currency; period average.

6/ There is a break is series beginning in 1998 when a new clastifieation of fiscal account was implemented.

$7 /$ Including the diccrepancy between monetary and fiscal data. 
Table 1. Lithuania: Key Economic and Financial Indicators, 1998-2002

\begin{tabular}{|c|c|c|c|c|c|}
\hline & 1998 & 1999 & 2000 & 2001 & 2002 \\
\hline & \multicolumn{5}{|c|}{ (Percentage changes from previous period) } \\
\hline Real GDP & 7.3 & -1.8 & 4.0 & 6.5 & 6.7 \\
\hline \multicolumn{6}{|l|}{ Consumer prices } \\
\hline Period average & 5.1 & 0.8 & 1.0 & 1.3 & 0.3 \\
\hline End of period & 2.4 & 0.3 & 1.5 & 2.0 & -1.0 \\
\hline Broad money & 14.5 & 7.7 & 16.5 & 21.4 & 16.9 \\
\hline Reserve money & 28.8 & -4.0 & -3.3 & 8.3 & 20.8 \\
\hline Credit to private sector & 16.9 & 13.8 & -1.2 & 15.4 & 30.4 \\
\hline Currency in circulation & 10.5 & -2.2 & -2.9 & 9.8 & 28.7 \\
\hline Velocity of broad money (level) & 5.7 & 4.9 & 4.7 & 4.2 & 3.8 \\
\hline Money multiplier (level) & 2.0 & 2.2 & 2.6 & 3.0 & 2.9 \\
\hline Deposit interest rate $1 /$ & 6.5 & 7.4 & 6.7 & 4.6 & 2.7 \\
\hline Lending interest rate l/ & 13.5 & 13.9 & 13.1 & 9.2 & 7.0 \\
\hline & \multicolumn{5}{|c|}{ (In percent of GDP) } \\
\hline Gross national saving & 12.3 & 11.9 & 14.2 & 16.2 & 17.4 \\
\hline General government & -1.1 & -3.9 & -0.2 & 0.1 & 1.7 \\
\hline Non-government & 13.4 & 15.9 & 14.3 & 16.1 & 15.6 \\
\hline Gross investment & 24.4 & 23.1 & 20.2 & 21.0 & 22.6 \\
\hline General government & 3.3 & 2.2 & 2.0 & 1.9 & 3.1 \\
\hline Non-government & 21.1 & 20.9 & 18.2 & 19.1 & 19.5 \\
\hline General government financial balance $2 /$ & -4.4 & -6.2 & -2.1 & -1.8 & -1.4 \\
\hline General government net lending & 1.5 & 2.4 & 0.7 & 0.2 & -0.2 \\
\hline Consolidated general government fiscal balance $2 /$ & -5.9 & -8.5 & -2.8 & -2.0 & -1.2 \\
\hline Extermal current account (incl. transfers) & -11.9 & -11.2 & -6.0 & -4.8 & -5.3 \\
\hline External debt $3 /$ & 34.4 & 42.5 & 43.5 & 44.4 & 44.5 \\
\hline \multicolumn{6}{|l|}{ Debt service (in percent of exports of } \\
\hline \multirow[t]{2}{*}{ goods and services) } & 22.6 & 18.5 & 20.9 & 35.1 & 48.6 \\
\hline & \multicolumn{5}{|c|}{ (In millions of litai at current prices) } \\
\hline Gross domestic product & 43,555 & 42,608 & 44,698 & 47,498 & 50,679 \\
\hline & \multicolumn{5}{|c|}{ (In millions of U.S. dollars) } \\
\hline Gross domestic product & 10,889 & 10,652 & 11,174 & 11,874 & 13,927 \\
\hline Current account balance & $-1,298$ & $-1,194$ & -675 & -574 & -734 \\
\hline
\end{tabular}

Sources: Department of Statistics, Bank of Lithuania, and Fund staff estimates and projections.

1/ In percent; weighted average of rates at commercial banks; 1-3 months' maturity.

2/ Inchudes discrepancy between above and below the line estimates of the financial balance and balances of budgetary organizations not recorded above the line. Also includes savings restitution payments.

3/ External liabilities minus equity investment. 
Table 2. Lithuania: Gross Value Added by Sector at Current Prices, 1998-2002

\begin{tabular}{|c|c|c|c|c|c|}
\hline & 1998 & 1999 & 2000 & 2001 & $20021 /$ \\
\hline & \multicolumn{4}{|c|}{ (In millions of Litai) } & \\
\hline Agriculture, hunting and forestry & 3,818 & 3,192 & 3,174 & 3,040 & 3,189 \\
\hline Fishing & 24 & 23 & 27 & 29 & 30 \\
\hline Mining and quarrying & 159 & 179 & 279 & 318 & 288 \\
\hline Manufacturing & 7,305 & 6,985 & 7,982 & 8,768 & 8,867 \\
\hline Electricity, gas and water supply & 1,639 & 1,660 & 1,541 & 1,786 & 1,911 \\
\hline Construction & 3,292 & 2,949 & 2,430 & 2,597 & 2,964 \\
\hline Wholesale and retail trade & 6,435 & 6,066 & 6,685 & 7,478 & 8,230 \\
\hline Hotels and restaurants & 630 & 652 & 619 & 677 & 742 \\
\hline Transport, storage and communications & 3,776 & 4,004 & 5,013 & 5,385 & 6,241 \\
\hline Financial intermediation & 859 & 868 & 881 & 988 & 1,065 \\
\hline Real estate, renting and business activities & 2,828 & 3,186 & 3,404 & 3,555 & 3,711 \\
\hline Public administration and defense & 2,679 & 2,678 & 2,787 & 2,475 & 2,497 \\
\hline Education & 2,381 & 2,655 & 2,596 & 2,723 & 2,808 \\
\hline Health and social work & 1,518 & 1,543 & 1,466 & 1,465 & 1,559 \\
\hline Other community services & 1,106 & 1,238 & 1,303 & 1,404 & 1,465 \\
\hline Private households with employed persons & 22 & 31 & 38 & 44 & 55 \\
\hline FISIM 2/ & 497 & 613 & 591 & 593 & -646 \\
\hline Gross Value Added Excluding FISIM & 37,973 & 37,297 & 39,633 & 42,135 & 44,975 \\
\hline Taxes less subsidies on products & 5,582 & 5,312 & 5,065 & 5,363 & 5,703 \\
\hline \multirow[t]{2}{*}{ Gross Domestic Product } & 43,555 & 42,608 & 44,698 & 47,498 & 50,679 \\
\hline & \multicolumn{5}{|c|}{ (In percent of GDP) } \\
\hline Agriculture, hunting and forestry & 9.9 & 8.4 & 7.9 & 7.1 & 7.0 \\
\hline Fishing & 0.1 & 0.1 & 0.1 & 0.1 & 0.1 \\
\hline Mining and quarrying & 0.4 & 0.5 & 0.7 & 0.7 & 0.6 \\
\hline Manufacturing & 19.0 & 18.4 & 19.8 & 20.5 & 19.4 \\
\hline Electricity, gas and water supply & 4.3 & 4.4 & 3.8 & 4.2 & 4.2 \\
\hline Construction & 8.6 & 7.8 & 6.0 & 6.1 & 6.5 \\
\hline Wholesale and retail trade & 16.7 & 16.0 & 16.6 & 17.5 & 18.0 \\
\hline Hotels and restaurants & 1.6 & 1.7 & 1.5 & 1.6 & 1.6 \\
\hline Transport, storage and communications & 9.8 & 10.6 & 12.5 & 12.6 & 13.7 \\
\hline Financial intermediation & 2.2 & 2.3 & 2.2 & 2.3 & 2.3 \\
\hline Real estate, renting and business activities & 7.4 & 8.4 & 8.5 & 8.3 & 8.1 \\
\hline Public administration and defense & 7.0 & 7.1 & 6.9 & 5.8 & 5.5 \\
\hline Education & 6.2 & 7.0 & 6.5 & 6.4 & 6.2 \\
\hline Health and social work & 3.9 & 4.1 & 3.6 & 3.4 & 3.4 \\
\hline Other community services & 2.9 & 3.3 & 3.2 & 3.3 & 3.2 \\
\hline
\end{tabular}

Source: Statistics Lithuania.

1/ Provisional data.

2/Financial intermediation services indirectly measured. 
Table 3. Lithuania: Gross Value Added by Sector at 2000 Constant Prices, 1998-2002

\begin{tabular}{|c|c|c|c|c|c|}
\hline & 1998 & 1999 & 2000 & 2001 & $20021 /$ \\
\hline & \multicolumn{5}{|c|}{ (In millions of Litai) } \\
\hline Agriculture, hunting and forestry & 3,488 & 2,983 & 3,174 & 3,027 & 3,213 \\
\hline Fishing & 21 & 24 & 27 & 27 & 27 \\
\hline Mining and quarying & 265 & 235 & 279 & 364 & 346 \\
\hline Manufacturing & 7,689 & 7,271 & 7,982 & 9,024 & 9,518 \\
\hline Electricity, gas and water supply & 1,797 & 1,797 & 1,541 & 1,773 & 1,870 \\
\hline Construction & 3,388 & 2,971 & 2,430 & 2,611 & 2,946 \\
\hline Wholesale and retail trade & 6,148 & 5,977 & 6,685 & 7,456 & 8,107 \\
\hline Hotels and restaurants & 677 & 660 & 619 & 625 & 673 \\
\hline Transport, storage and communications & 4,911 & 4,903 & 5,013 & 5,234 & 5,893 \\
\hline Financial intermediation & 859 & 884 & 881 & 921 & 1,018 \\
\hline Real estate, renting and business activities & 2,867 & 3,165 & 3,404 & 3,653 & 3,753 \\
\hline Public administration and defense & 2,423 & 2,571 & 2,787 & 2,653 & 2,771 \\
\hline Education & 2,242 & 2,440 & 2,596 & 2,649 & 2,656 \\
\hline Health and social work & 1,403 & 1,501 & 1,466 & 1,454 & 1,497 \\
\hline Other community services & 1,212 & 1,308 & 1,303 & 1,316 & 1,353 \\
\hline Private households with employed persons & 26 & 33 & 38 & 47 & 56 \\
\hline FISIM $2 /$ & 590 & 606 & 591 & 616 & 649 \\
\hline Gross Value Added Excluding FISIM & 38,825 & 38,117 & 39,633 & 42,216 & 45,049 \\
\hline Taxes less subsidies on products & 4,961 & 4,871 & 5,065 & 5,395 & 5,757 \\
\hline \multirow[t]{2}{*}{ Gross Domestic Product } & 43,786 & 42,988 & 44,698 & 47,611 & 50,805 \\
\hline & \multicolumn{5}{|c|}{ (In percent of GDP) } \\
\hline Agriculture, hunting and forestry & 8.0 & 6.9 & 7.1 & 6.4 & 6.3 \\
\hline Fishing & 0.0 & 0.1 & 0.1 & 0.1 & 0.1 \\
\hline Mining and quanrying & 0.6 & 0.5 & 0.6 & 0.8 & 0.7 \\
\hline Manufacturing & 17.6 & 16.9 & 17.9 & 19.0 & 18.7 \\
\hline Electricity, gas and water supply & 4.1 & 4.2 & 3.4 & 3.7 & 3.7 \\
\hline Construction & 7.7 & 6.9 & 5.4 & 5.5 & 5.8 \\
\hline Wholesale and retail trade & 14.0 & 13.9 & 15.0 & 15.7 & 16.0 \\
\hline Hotels and restaurants & 1.5 & 1.5 & 1.4 & 1.3 & 1.3 \\
\hline Transport, storage and conmmunications & 11.2 & 11.4 & 11.2 & 11.0 & 11.6 \\
\hline Financial intermediation & 2.0 & 2.1 & 2.0 & 1.9 & 2.0 \\
\hline Real estate, renting and business activities & 6.5 & 7.4 & 7.6 & 7.7 & 7.4 \\
\hline Public administration and defense & 5.5 & 6.0 & 6.2 & 5.6 & 5.5 \\
\hline Education & 5.1 & 5.7 & 5.8 & 5.6 & 5.2 \\
\hline Health and social work & 3.2 & 3.5 & 3.3 & 3.1 & 2.9 \\
\hline Other community services & 2.8 & 3.0 & 2.9 & 2.8 & 2.7 \\
\hline
\end{tabular}

Source: Statistics Lithuania.

1/ Provisional data.

2/Financial intermediation services indirectly measured. 
Table 4. Lithuania: Gross Domestic Product by Expenditure at Current Prices, 1998-2002

\begin{tabular}{|c|c|c|c|c|c|}
\hline & 1998 & 1999 & 2000 & 2001 & $20021 /$ \\
\hline & \multicolumn{5}{|c|}{ (In millions of Litai) } \\
\hline Gross domestic product & 43,555 & 42,608 & 44,698 & 47,498 & 50,679 \\
\hline Domestic Demand & 48,665 & 47,005 & 47,593 & 50,104 & 53,248 \\
\hline Final consumption expenditure & 37,198 & 37,147 & 38,567 & 40,124 & 41,862 \\
\hline Househoids consumption expenditure & 26,396 & 27,442 & 28,581 & 30,407 & 31,385 \\
\hline Government consumption expenditure & 10,767 & 9,634 & 9,854 & 9,598 & 10,358 \\
\hline NPI serving househoids & 35 & 71 & 132 & 119 & 119 \\
\hline Gross domestic investment & 11,467 & 9,858 & 9,026 & 9,980 & 11,386 \\
\hline Gross fixed capital formation & 10,723 & 9,614 & 8,565 & 9,785 & 10,906 \\
\hline Changes in stocks & 731 & 229 & 449 & 186 & 486 \\
\hline Trade Balance & $-5,110$ & $-4,397$ & $-2,894$ & $-2,607$ & $-2,570$ \\
\hline Exports of goods and services & 20,283 & 16,953 & 20,437 & 24,182 & 27,391 \\
\hline \multirow[t]{2}{*}{ Imports of goods and services } & 25,393 & 21,350 & 23,331 & 26,789 & 29,961 \\
\hline & \multicolumn{5}{|c|}{ (In percent of GDP) } \\
\hline Domestic Demand & 111.7 & 110.3 & 106.5 & 105.5 & 105.1 \\
\hline Final consumption expenditure & 85.4 & 87.2 & 86.3 & 84.5 & 82.6 \\
\hline Households consumption expenditure & 60.6 & 64.4 & 63.9 & 64.0 & 61.9 \\
\hline Government consumption expenditure & 24.7 & 22.6 & 22.0 & 20.2 & 20.4 \\
\hline NPI serving households & 0.1 & 0.2 & 0.3 & 0.3 & 0.2 \\
\hline Gross domestic investment & 26.3 & 23.1 & 20.2 & 21.0 & 22.6 \\
\hline Gross fixed capital formation & 24.6 & 22.6 & 19.2 & 20.6 & 21.5 \\
\hline Changes in stocks & 1.7 & 0.5 & 1.0 & 0.4 & 1.0 \\
\hline Trade Balance & -11.7 & -10.3 & -6.5 & -5.5 & -5.7 \\
\hline Exports of goods and services & 46.6 & 39.8 & 45.7 & 50.9 & 54.0 \\
\hline Imports of goods and services & 58.3 & 50.1 & 52.2 & 56.4 & 59.1 \\
\hline
\end{tabular}

Source: Statistics Lithuania.

1/ Provisional data. 
Table 5. Lithuania: Gross Domestic Product by Expenditure at 2000 Constant Prices, 1998-2002

\begin{tabular}{|c|c|c|c|c|c|}
\hline & 1998 & 1999 & 2000 & 2001 & $20021 /$ \\
\hline & \multicolumn{5}{|c|}{ (In millions of Litai) } \\
\hline Gross domestic product & 43,786 & 42,988 & 44,698 & 47,611 & 50,805 \\
\hline Domestic Demand & 46,848 & 46,650 & 47,593 & 50,289 & 53,106 \\
\hline Final consumption expenditure & 36,392 & 36,411 & 38,567 & 39,728 & 41,415 \\
\hline Households consumption expenditure & 26,032 & 26,856 & 28,581 & 29,721 & 30,981 \\
\hline Government consumption expenditure & 10,324 & 9,484 & 9,854 & 9,888 & 10,315 \\
\hline Gross domestic investment & 10,456 & 10,239 & 9,026 & 10,561 & 11,691 \\
\hline Gross fixed capital formation & 10,025 & 9,410 & 8,565 & 9,724 & 10,930 \\
\hline Changes in stocks & 418 & 814 & 449 & 829 & 767 \\
\hline Trade Balance & $-3,061$ & $-3,662$ & $-2,894$ & $-2,678$ & $-2,301$ \\
\hline Exports of goods and services & 22,370 & 18,616 & 20,437 & 24,775 & 29,578 \\
\hline \multirow[t]{2}{*}{ Imports of goods and services } & 25,431 & 22,278 & 23,331 & 27,453 & 31,879 \\
\hline & \multicolumn{5}{|c|}{ (In percent of GDP) } \\
\hline Domestic Demand & 107.0 & 108.5 & 106.5 & 105.6 & 104.5 \\
\hline Final consumption expenditure & 83.1 & 84.7 & 86.3 & 83.4 & 81.5 \\
\hline Households consumption expenditure & 59.5 & 62.5 & 63.9 & 62.4 & 61.0 \\
\hline Government consumption expenditure & 23.6 & 22.1 & 22.0 & 20.8 & 20.3 \\
\hline Gross domestic investment & 23.9 & 23.8 & 20.2 & 22.2 & 23.0 \\
\hline Gross fixed capital formation & 22.9 & 21.9 & 19.2 & 20.4 & 21.5 \\
\hline Changes in stocks & 1.0 & 1.9 & 1.0 & 1.7 & 1.5 \\
\hline Trade Balance & -7.0 & -8.5 & -6.5 & -5.7 & -4.5 \\
\hline Exports of goods and services & 51.1 & 43.3 & 45.7 & 52.0 & 58.2 \\
\hline Imports of goods and services & 58.1 & 51.8 & 52.2 & 57.7 & 62.7 \\
\hline
\end{tabular}

Source: Statistics Lithuania.

1/ Provisional data. 
Table 6. Lithuania: Employment and Wages, 1998-2002

\begin{tabular}{|c|c|c|c|c|c|}
\hline & 1998 & 1999 & 2000 & 2001 & $2002 \mathrm{l} /$ \\
\hline Total Employed, thousands $2 /$ & 1,656 & 1,648 & 1,586 & 1,352 & 1,406 \\
\hline By the public sector & 528 & 512 & 495 & 453 & 423 \\
\hline By the private sector & 1,129 & 1,136 & 1,091 & 899 & 983 \\
\hline Total labor force, thousands $2 /$ & 1,770 & 1,796 & 1,791 & 1,636 & 1,630 \\
\hline Total participation rate, in percent $3 /$ & 61.3 & 61.9 & 61.4 & 69.4 & 69.3 \\
\hline \multicolumn{6}{|l|}{ Employed by sector, thousands $2 /$} \\
\hline Agriculture & 356 & 333 & 315 & 234 & 251 \\
\hline Industry & 331 & 326 & 320 & 366 & 387 \\
\hline Services & 969 & 989 & 951 & 752 & 768 \\
\hline Total average gross wage, local currency, nominal & 930 & 987 & 971 & 982 & 1,034 \\
\hline Public sector & 1,033 & 1,090 & 1,077 & 1,098 & 1,133 \\
\hline Private sector & 840 & 894 & 878 & 888 & 957 \\
\hline Minimum wage & 418 & 430 & 430 & 430 & 430 \\
\hline
\end{tabular}

Source: Statistics Lithuania.

1/ Provisional data.

2/ Figures for 2001 and 2002 reflect a downward revision of the estimated population.

3 / Residents aged 15-64; in earlier years those aged 16 and above. 


$$
-10-
$$

Table 7. Lithuania: Unemployment, 1998-2002

\begin{tabular}{|c|c|c|c|c|c|}
\hline & 1998 & 1999 & 2000 & 2001 & 2002 \\
\hline \multicolumn{6}{|l|}{ Unemployment } \\
\hline by registration (average) & 6.4 & 8.4 & 11.5 & 12.5 & 11.3 \\
\hline by survey & 13.3 & 14.1 & 15.4 & 17.4 & 13.8 \\
\hline Unemployed by duration (end of the year, \%) & 100 & 100 & 100 & 100 & 100 \\
\hline 6 months and below & 27.2 & 36.7 & 33.0 & 20.2 & 27.5 \\
\hline 7 to 12 months & 17.8 & 24.2 & 14.9 & 21.0 & 17.6 \\
\hline 1 year and above & 55.0 & 39.1 & 52.2 & 58.8 & 54.9 \\
\hline \multicolumn{6}{|l|}{ Education of the unemployed, in percent } \\
\hline Total & 100 & 100 & 100 & 100 & 100 \\
\hline Higher education & 6.9 & 7.9 & 7.2 & 6.7 & 7.4 \\
\hline College level & 20.1 & 20.6 & 21.7 & 22.0 & 22.5 \\
\hline Secondary without vocational training & 28.0 & 24.7 & 23.1 & 27.2 & 28.9 \\
\hline Secondary with vocational training & 20.1 & 22.7 & 22.2 & 21.3 & 18.4 \\
\hline Basic without vocational training & 13.6 & 13.3 & 14.9 & 13.6 & 15.1 \\
\hline Basic with vocational training & 8.2 & 8.7 & 8.7 & 6.6 & 5.8 \\
\hline Primary & 3.1 & 2.1 & 2.1 & 2.6 & 1.9 \\
\hline \multicolumn{6}{|c|}{ Unemployment rate of the population by counties (by survey) } \\
\hline \multicolumn{6}{|l|}{ Provinces } \\
\hline Alytus & 18.8 & 21.1 & 16.3 & 22.6 & 16.2 \\
\hline Kaunes & 11.5 & 13.5 & 15.0 & 17.8 & 14.0 \\
\hline Klaipeda & 10.8 & 14.9 & 14.4 & 17.0 & 12.3 \\
\hline Marijampole & 11.8 & 13.5 & 15.5 & 18.5 & 10.5 \\
\hline Panevezys & 14.1 & 12.4 & 14.6 & 14.6 & 13.3 \\
\hline Siauliai & 14.7 & 16.8 & 18.4 & 19.5 & 14.8 \\
\hline Taurage & 10.8 & 12.6 & 13.7 & 16.4 & 8.6 \\
\hline Telsiai & 9.9 & 10.5 & 14.0 & 18.6 & 15.4 \\
\hline Utena & 13.9 & 11.1 & 13.6 & 16.6 & 14.3 \\
\hline Vilnius & 14.8 & 13.9 & 15.7 & 16.0 & J4.5 \\
\hline Total, whole country & 13.3 & 14.1 & 15.4 & 17.4 & 33.8 \\
\hline
\end{tabular}

Source: Statisties Lithuania. 
Table 8. Lithuania: Major Privatization Transactions, 1998-2002

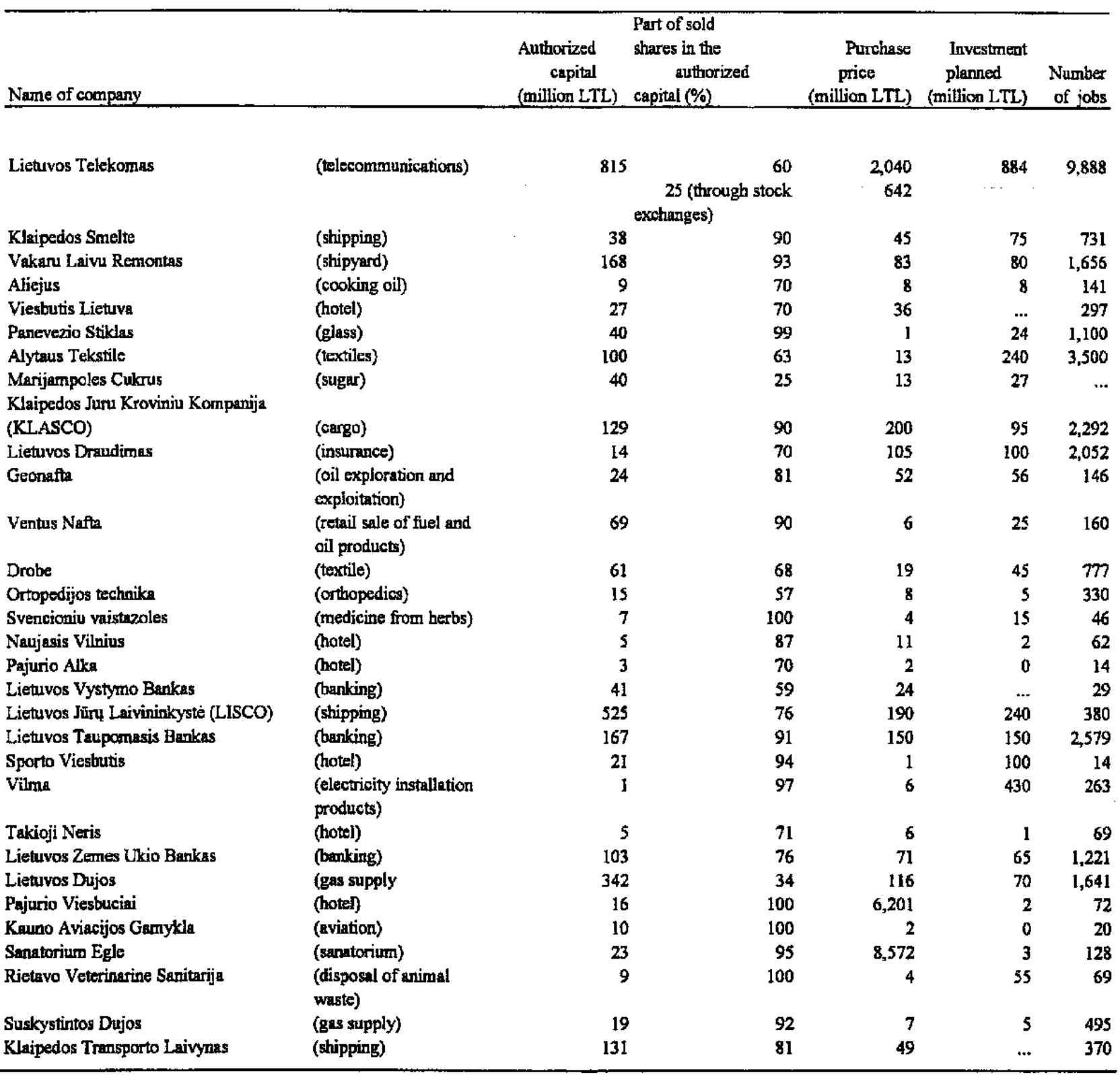

Source: Stste Property Fund. 


$$
-12-
$$

Table 9. Lithuania: Bankruptcy Cases, 1998-2002

\begin{tabular}{|c|c|c|c|c|c|c|}
\hline & 1998 & 1999 & 2000 & 2001 & 2002 & Total \\
\hline \multicolumn{7}{|l|}{ Bankruptcy petitions to courts } \\
\hline Bankruptcy cases initiated & 97 & 243 & 402 & 578 & 787 & 2,107 \\
\hline of which: Bankruptcy cases finalized & 10 & 183 & 261 & 205 & 47 & 706 \\
\hline \multicolumn{7}{|l|}{ Out-of-court proceedings } \\
\hline Bankruptcy cases initiated & 2 & 4 & 12 & 14 & 11 & 43 \\
\hline of which: Bankruptcy cases finalized & 1 & 4 & 10 & 11 & 2 & 28 \\
\hline \multicolumn{7}{|l|}{ Total } \\
\hline Bankruptcy cases initiated & 98 & 247 & 414 & 592 & 798 & 2,149 \\
\hline of which: Banknuptcy cases finalized & 74 & 183 & 263 & 209 & 507 & 1,236 \\
\hline
\end{tabular}

Source: Ministry of Economy. 
Table 10: Lithuania: Balance of Payments, 1998-2002

\begin{tabular}{|c|c|c|c|c|c|}
\hline & 1998 & 1999 & 2000 & 2001 & 2002 \\
\hline & \multicolumn{5}{|c|}{ (In millions of U.S. dollars, uniess otherwise indicated) } \\
\hline Current accoungt & $-1,298$ & $-1,194$ & -675 & -573 & -734 \\
\hline Trade baiance & $-1,518$ & $-1,405$ & $-1,104$ & $-1,108$ & $-1,337$ \\
\hline Exports (f. o.b.) & 3,962 & 3,147 & 4,050 & 4,889 & 6,031 \\
\hline Imports (f.o.b.) & 5,480 & 4,551 & 5,154 & 5,997 & 7,368 \\
\hline Non-faxtor services, net & 241 & 305 & 380 & 457 & 544 \\
\hline Creatis & 1,109 & 1,092 & 1,059 & 1,157 & 1,479 \\
\hline Debita & 868 & 786 & 679 & 700 & 935 \\
\hline Factor income, net & .255 & -258 & -194 & -180 & -174 \\
\hline Current transfern, set & 235 & 163 & 243 & 258 & 233 \\
\hline Capital and fmmeial account & 1,444 & 1,047 & 679 & 745 & 1,014 \\
\hline Capital transfiers, net & -2 & -3 & 2 & 1 & 57 \\
\hline Financiel account & 1,446 & 1,050 & 677 & 744 & 957 \\
\hline Diacet imvestnent, net & 921 & 478 & 375 & 439 & 714 \\
\hline Pontfolio investomant, net & -20 & 511 & 265 & 264 & -3 \\
\hline Other inventoment, at & 545 & 61 & 37 & 41 & 246 \\
\hline Net erron and amionions & 253 & -48 & 127 & 153 & 143 \\
\hline Firnencing & -429 & 179 & -157 & -359 & -463 \\
\hline Fund financing & -30 & -17 & -26 & -34 & -40 \\
\hline Cunge in official reserves (-increase) & -399 & 196 & -131 & -325 & -423 \\
\hline \multicolumn{6}{|l|}{ Official renerves and exteral debt } \\
\hline Growa official relerven $\mathbf{l}$ & 1,460 & 1,242 & 1,359 & 1,669 & 2,420 \\
\hline Grow extemal debt $2 /$ & 3,741 & 4,528 & 4,856 & 5,268 & 6,199 \\
\hline Public and publicly gurarentred & 1,670 & 2,383 & 2,364 & 2,332 & 2,429 \\
\hline Private & 2,071 & 2,145 & 2,493 & 2,936 & 3,770 \\
\hline \multicolumn{6}{|l|}{ Short-derm Groan external debt } \\
\hline On a remsining maturity baris & 1,730 & 1,899 & 2,786 & 4,315 & 4,636 \\
\hline On an original maturity baris & 1,042 & 1,045 & 1,114 & 1,558 & 2,123 \\
\hline Debt vervice $3 /$ & 1,144 & 846 & 1,066 & 2,123 & 2,936 \\
\hline & \multicolumn{5}{|c|}{ (In percent of GDP) } \\
\hline Current accourat & -11.9 & -11.2 & -6.0 & -4.8 & -5.3 \\
\hline Trade balunce of goods and services & -11.7 & -10.3 & -6.5 & .5 .5 & -5.7 \\
\hline Trade baltance, goods & -13.9 & -13.2 & -9.9 & -9.3 & -9.6 \\
\hline Trade balance, services & 2.2 & 2.9 & 3.4 & 3.8 & 3.9 \\
\hline Factor income, net & -2.3 & -2.4 & -1.7 & -1.5 & -1.3 \\
\hline Current tranafen, net & 2.2 & 1.5 & 2.2 & 2.2 & 1.7 \\
\hline Finnacial eccoumt & 13.3 & 9.9 & 6.1 & 6.3 & 6.9 \\
\hline Diroct inverement, net & 8.5 & 4.5 & 3.4 & 3.7 & 5.1 \\
\hline Portfolio inveituent, net & -0.2 & 4.8 & 2.4 & 2.2 & 0.0 \\
\hline Other inveriment, net & 5.0 & 0.6 & 0.3 & 0.3 & 1.8 \\
\hline Grow excemil debt 2 & 34.4 & 42,5 & 43.5 & 44.4 & 44.7 \\
\hline Public and publichy guaranteed & 15.3 & 22.4 & 21.2 & 19.6 & 17.5 \\
\hline Privale & 19.0 & 20.1 & 22.3 & 24.7 & 27.2 \\
\hline \multicolumn{6}{|l|}{ Shont-term Groes exkerni debx } \\
\hline On a rempining meturity berin & 16.1 & 17.8 & 24.9 & 36.3 & 33.3 \\
\hline On an cuiginal maturity basia & 9.7 & 9.8 & 9.8 & 13.1 & 15.2 \\
\hline Debt tervice, in percent of exports of GNFS $3 /$ & 22.6 & 20.0 & 20.9 & 17.9 & 21.1 \\
\hline \multicolumn{6}{|l|}{ Memonandum items: } \\
\hline Noarint GDP (mitions of U.S. dolars) & 10,889 & 10,652 & 11,174 & 11,874 & 13,927 \\
\hline Exports of GNFS (percent changf, $y-0-y$ ) & -2.9 & -16.4 & 20.5 & 18.3 & 33.4 \\
\hline Import of GNFS (percedt change, $y-0-y$ ) & 1.8 & -15.9 & 9.3 & 14.8 & 13.1 \\
\hline
\end{tabular}


Table 11. Lithuania: Merchandise Trade by Commodity Group, 1998-2002

(In units as indicated)

\begin{tabular}{|c|c|c|c|c|c|}
\hline & 1998 & 1999 & 2000 & 2001 & 2002 \\
\hline \multicolumn{6}{|l|}{ Exports } \\
\hline Total (in millions of U.S. dollars) & 3,962 & 3,147 & 4,050 & 4,889 & 6,031 \\
\hline & \multicolumn{5}{|c|}{ (In percent of total) } \\
\hline Animal and vegetable products & 9.6 & 8.0 & 7.4 & 7.6 & 6.1 \\
\hline Food, beverages and tobacco & 4.4 & 4.4 & 4.3 & 4.8 & 4.5 \\
\hline Mineral products & 19.2 & 15.1 & 21.2 & 23.4 & 18.9 \\
\hline Chemical products & 9.6 & 9.4 & 7.9 & 6.4 & 6.4 \\
\hline Plastic and rubber products & 2.4 & 2.8 & 3.1 & 2.9 & 2.8 \\
\hline Rawhide, skins and leather products & 1.4 & 1.7 & 1.4 & 1.2 & 1.0 \\
\hline Wood and wood products & 4.8 & 6.4 & 5.9 & 5.3 & 5.4 \\
\hline Pulp and paper & 1.7 & 1.5 & 1.2 & 1.3 & 1.3 \\
\hline Textiles and footwear & 19.1 & 23.4 & 19.0 & 16.7 & 15.4 \\
\hline Cement, glass and similar materials & 1.3 & 1.3 & 1.2 & 1.1 & 1.0 \\
\hline Base metals and products of base metals & 3.8 & 3.6 & 4.2 & 3.7 & 4.7 \\
\hline Machinery, mechsnical appliances and electrical equipment & 10.8 & 11.4 & 10.6 & 10.7 & 10.0 \\
\hline Vehicles and transport equipment & 8.1 & 5.4 & 6.8 & 9.2 & 16.0 \\
\hline Optical, photographic, and measuring instruments & 1.0 & 1.1 & 1.1 & 1.1 & 1.0 \\
\hline Other $1 /$ & 2.7 & 4.1 & 4.4 & 4.3 & 5.1 \\
\hline \multicolumn{6}{|l|}{ Imports } \\
\hline \multirow[t]{2}{*}{ Total (in millions of U.S. dollars) } & 5,480 & 4,551 & 5,154 & 5,997 & 7,368 \\
\hline & \multicolumn{5}{|c|}{ (In percent of total) } \\
\hline Animal and vegetable products & 6.1 & 5.9 & 6.1 & 5.7 & 4.7 \\
\hline Food, beverages and tobacco & 4.8 & 5.4 & 4.0 & 3.9 & 3.5 \\
\hline Mineral products & 15.6 & 16.6 & 23.3 & 21.2 & 17.6 \\
\hline Chemical products & 9.2 & 10.0 & 9.1 & 9.2 & 8.6 \\
\hline Plastic and rubber products & 5.1 & 5.3 & 5.5 & 5.3 & 5.2 \\
\hline Rawhide, skins and leather products & 0.7 & 0.9 & 0.7 & 0.8 & 0.8 \\
\hline Wood and wood products & 1.1 & 1.3 & 1.3 & 1.3 & 1.4 \\
\hline Pulp and paper & 3.4 & 3.6 & 2.9 & 2.7 & 2.5 \\
\hline Textiles and footwear & 9.5 & 10.9 & 9.8 & 9.4 & 8.5 \\
\hline Cement, glass and similar materials & 1.7 & 1.8 & 1.6 & 1.6 & 1.5 \\
\hline Base metals and products of base metals & 6.2 & 5.5 & 5.3 & 5.2 & 6.7 \\
\hline Machinery, mechanical appliances and electrical equipment & 18.4 & 18.4 & 15.7 & 16.7 & 17.3 \\
\hline Vehicles and transport equipment & 12.3 & 7.5 & 8.7 & 11.5 & 16.2 \\
\hline Optical, photographic, and measuring instruments & 2.3 & 2.5 & 1.6 & 1.7 & 1.6 \\
\hline Other 1/ & 3.5 & 4.2 & 4.3 & 3.7 & 3.5 \\
\hline
\end{tabular}

Source: Statistics Lithuania.

1/ Includes precious stones and metals, arm and ammunitions, works of art, and miscellaneous manufactured articles. 
Table 12. Lithuania: Merchandise Trade by Country and Country Group, 1998-2002

(In units as indicated)

\begin{tabular}{|c|c|c|c|c|c|c|c|c|c|c|}
\hline & \multicolumn{5}{|c|}{ Exports } & \multicolumn{5}{|c|}{ Imports 1/ } \\
\hline & 1998 & 1999 & 2000 & 2001 & 2002 & 1998 & 1999 & 2000 & 2001 & 2002 \\
\hline \multirow[t]{2}{*}{ Total (in millions of U.S. dollan) } & 3,962 & 3,147 & 4,050 & 4,889 & 6,031 & 5,480 & 4,551 & 5,154 & 5,997 & 7,368 \\
\hline & \multicolumn{10}{|c|}{ (In percent) } \\
\hline EU & 38.0 & 50.1 & 47.9 & 47.8 & 48.4 & 47.2 & 46.5 & 43.3 & 44.0 & 44.5 \\
\hline \multicolumn{11}{|l|}{ Ofwhich: } \\
\hline Belgium & 1.3 & 1.7 & 1.6 & 1.6 & 1.9 & 19 & 2.1 & 2.0 & 2.0 & 1.9 \\
\hline Denmark & 4.1 & 6.2 & 4.9 & 4.5 & 5.1 & 3.8 & 3.9 & 3.1 & 2.9 & 2.9 \\
\hline United Kingdom & 3.5 & 5.1 & 7.8 & 13.8 & 13.4 & 3.7 & 4.2 & 4.5 & 3.4 & 3.3 \\
\hline Italy & 4.1 & 4.2 & 2.3 & 2.0 & 2.8 & 4.4 & 4.1 & 3.6 & 4.2 & 4.9 \\
\hline Netherlands & 2.5 & 3.5 & 4.8 & 2.9 & 3.2 & 2.2 & 2.3 & 2.3 & 2.4 & 2.2 \\
\hline France & 3.5 & 4.7 & 4.4 & 3.3 & 4.1 & 3.4 & 3.6 & 4.2 & 3.8 & 3.9 \\
\hline Finland & 0.9 & 1.0 & 1.3 & 2.4 & 1.2 & 3.2 & 3.1 & 2.6 & 2.3 & 2.3 \\
\hline Sweden & 2.6 & 4.2 & 4.4 & 3.7 & 4.2 & 3.7 & 3.4 & 3.4 & 3.0 & 3.3 \\
\hline Germuny & I3.1 & 16.0 & 14.3 & 12.6 & 10.4 & 18.2 & 16.5 & 15.1 & 17.2 & 17.0 \\
\hline EFTA & 2.2 & 2.7 & 2.5 & 1.9 & 4.1 & 2.0 & 2.1 & 1.8 & 1.9 & 2.5 \\
\hline \multicolumn{11}{|l|}{ Of which: } \\
\hline Switzerland & 0.8 & 1.5 & 1.3 & 0.6 & 1.7 & 1.6 & 1.5 & 1.3 & 1.2 & 1.2 \\
\hline Norway & 0.5 & 1.1 & 1.1 & 1.3 & 24 & 0.8 & 0.7 & 0.8 & 1.0 & 1.5 \\
\hline CEFTA & 3.9 & 6.0 & 70 & 7.3 & 4.8 & 9.0 & 9.7 & 8.1 & 8.0 & 7.8 \\
\hline \multicolumn{11}{|l|}{ Of which: } \\
\hline Poland & 3.0 & 4.5 & 5.5 & 6.3 & 3.6 & 5.5 & 5.7 & 4.9 & 4.9 & 4.8 \\
\hline Czech Republic & 0.5 & 0.8 & 1.0 & 0.5 & 0.4 & 1.7 & 1.8 & 1.4 & 1.4 & 1.3 \\
\hline CIS & 35.7 & 18.2 & 16.3 & 19,7 & 19.2 & 26.0 & 24.4 & 31.7 & 29.4 & 26.0 \\
\hline \multicolumn{11}{|l|}{ Of which: } \\
\hline Belarus & 8.8 & 5.9 & 29 & 3.9 & 3.2 & 2.2 & 2.2 & 1.8 & 1.9 & 1.5 \\
\hline Ronie & 16.5 & 7.0 & 7.1 & 11.0 & 12.2 & 21.2 & 20.1 & 27.4 & 25.3 & 21.3 \\
\hline Uthrins & 7.8 & 3.7 & 4.4 & 3.4 & 2.6 & 1.9 & 1.5 & 1.5 & 1.6 & 1.6 \\
\hline Othern & 20.2 & 23.0 & 26.3 & 23.3 & 23.5 & 12.6 & 13.6 & 11.2 & 12.3 & 14.0 \\
\hline \multicolumn{11}{|l|}{ Of which: } \\
\hline Eutania & 2.6 & 2.4 & 23 & 3.2 & 3.8 & 2.7 & 2.6 & 2.3 & 24 & 3.0 \\
\hline Latvia & 11.1 & 12.8 & 15.0 & 12.6 & 9.6 & 1.8 & 2.0 & 1.6 & 1.5 & 1.6 \\
\hline Japan & 0.3 & 0.3 & 0.3 & 0.4 & 0.2 & 2.0 & 1.6 & 1.9 & 1.9 & 2.1 \\
\hline USA & 2.8 & 4.4 & 4.9 & 3.8 & 3.5 & 2.9 & 3.8 & 24 & 3.0 & 2.8 \\
\hline Cunada & 0.1 & 0.1 & 0.3 & 0.3 & 0.5 & 0.1 & 0.1 & 0.1 & 0.1 & 0.1 \\
\hline Turkey & 0.9 & 1.0 & 1.8 & 1.4 & 1.7 & 0.3 & 0.3 & 0.2 & 0.3 & 0.5 \\
\hline
\end{tabular}

Source: Statistice Lithuanis.

$1 /$ imported goode by country are broken up acsording the country of consigument. 
$-16-$

Table 13. Lithuania: Summary of Import Tariff Rates, June 2003

\begin{tabular}{|c|c|c|c|c|c|c|c|c|c|c|c|c|c|}
\hline \multirow{5}{*}{ No } & \multirow{5}{*}{ Turiff } & \multicolumn{12}{|c|}{ Position numban to which Cutom tariff are xpplied } \\
\hline & & \multicolumn{6}{|c|}{ Antonomoms } & \multicolumn{6}{|c|}{ Conventional } \\
\hline & & \multirow{2}{*}{\multicolumn{2}{|c|}{ Lotal }} & \multicolumn{4}{|c|}{ of that number } & \multirow{2}{*}{\multicolumn{2}{|c|}{ total }} & \multicolumn{4}{|c|}{ of that numbor } \\
\hline & & & & \multicolumn{2}{|c|}{ Agricularal goods } & \multicolumn{2}{|c|}{ Induatrial goods } & & & \multicolumn{2}{|c|}{ Apricultural goods } & \multicolumn{2}{|c|}{ Induatrial goods } \\
\hline & & ch. $1-99$ & $\%$ & sh. $1-24$ & $\%$ & ch.25-99 & $\%$ & ch 1-99 & $\%$ & ch. $1-24$ & $\%$ & ch. $25-99$ & $\%$ \\
\hline 1 & 0 & 7,735 & 69.55 & 896 & 32.49 & 6,839 & 81.78 & 7,741 & 69.61 & 896 & 32.49 & 6,845 & 81.85 \\
\hline 2 & 3 & 0 & 0.00 & 0 & 0 & 0 & 0.00 & 1 & 0.01 & 1 & 0.04 & 0 & 0.00 \\
\hline 3 & 3.5 & 0 & 0.00 & 0 & 0 & 0 & 0.00 & 3 & 0.03 & 0 & 0 & 3 & 0.04 \\
\hline 4 & 4 & 0 & 0 & 0 & 0 & 0 & 0 & 2 & 0.02 & 0 & 0 & 2 & 0.02 \\
\hline 5 & 5 & 15 & 0.13 & 1 & 0.04 & 14 & 0.17 & 887 & 7.98 & 712 & 25.82 & 175 & 2.09 \\
\hline 6 & 6.5 & 2 & 0 & 0 & 0 & 2 & 0 & 85 & 0.76 & 0 & 0 & 85 & 1.02 \\
\hline 7 & 7 & 0 & 0.00 & 0 & 0.00 & 0 & 0.00 & 1 & 0.01 & 0 & 0.00 & 1 & 0.01 \\
\hline 8 & 7 & $\mathbf{0}$ & 0.00 & 0 & 0.00 & 0 & 0.00 & 103 & 0.93 & 101 & 3.66 & 2 & 0.02 \\
\hline 9 & 10 & 903 & 8.12 & 712 & 25.82 & 191 & 2.28 & 361 & 3.25 & 33 & 1.20 & 328 & 3.92 \\
\hline 10 & 12 & 0 & 0.00 & 0 & 0.00 & 0 & 0.00 & 77 & 0.69 & 0 & 0.00 & 77 & 0.92 \\
\hline 11 & 12 & 0 & 0.00 & 0 & 0.00 & 0 & 0.00 & 1 & 0.01 & 0 & 0.00 & 1 & 0 \\
\hline 12 & 13 & D & 0.00 & 0 & 0.00 & 0 & 0.00 & 29 & 0.26 & 0 & 0.00 & 29 & 0 \\
\hline 13 & 13 & 0 & 0.00 & 0 & 0.00 & 0 & 0 & 232 & 2.09 & 0 & 0.00 & 232 & 3 \\
\hline 14 & 13 & 0 & 0.00 & 0 & 0.00 & 0 & 0.00 & 29 & 0.26 & 0 & 0.00 & 29 & 0 \\
\hline 15 & 15 & 397 & 3.57 & 134 & 4.86 & 263 & $\mathbf{3}$ & 439 & 4 & 17 & 1 & 422 & 5 \\
\hline 16 & 17 & 0 & 0 & 0 & 0 & 0 & 0 & 23 & 0.21 & 0 & 0.00 & 23 & 0 \\
\hline 17 & 18 & 0 & 0.00 & 0 & 0.00 & 0 & 0 & 1 & 0 & 1 & 0 & 0 & 0 \\
\hline 18 & 19 & 0 & 0.00 & 0 & 0.00 & 0 & 0 & 10 & 0.09 & 10 & 0.36 & 0 & 0 \\
\hline 19 & 20 & 419 & 3.77 & 22 & 0.80 & 397 & 3 & 229 & 2 & 198 & 7 & 31 & 0 \\
\hline 20 & 22 & 0 & 0.00 & 0 & 0.00 & 0 & 0 & 10 & 0 & 0 & 0 & 10 & 0 \\
\hline 21 & 23 & 0 & 0.00 & 0 & 0.00 & 0 & 0 & 27 & 0 & 27 & ] & 0 & 0 \\
\hline 22 & 23 & 0 & 0.00 & 0 & 0.00 & 0 & 0 & 3 & 0 & 3 & 0 & 0 & 0 \\
\hline 23 & 23 & 0 & 0.00 & 0 & 0.00 & 0 & 0 & 4 & 0 & 4 & 0 & 0 & 0 \\
\hline 24 & 25 & 734 & 6.60 & 194 & 7.03 & 540 & 6 & 67 & 1 & 12 & 0 & 55 & 1 \\
\hline 25 & 26 & 0 & 0.00 & 0 & 0.00 & 0 & 0 & 30 & 0. & 30 & 1 & 0 & 0 \\
\hline 26 & 28 & 0 & 0.00 & 0 & 0.00 & 0 & 0 & 49 & 0 & 49 & 2 & 0 & 0 \\
\hline 27 & 28 & 0 & 0.00 & 0 & 0.00 & 0 & 0 & 10 & 0 & 10 & D & 0 & 0 \\
\hline 28 & 30 & 79 & 0.71 & 12 & 0.44 & 67 & 1 & 220 & 2 & 207 & 8 & 13 & 0 \\
\hline 29 & 33 & 0 & 0.00 & 0 & 0.00 & 0 & 0 & 88 & 1 & 88 & 3 & 0 & 0 \\
\hline 30 & 33 & 0 & 0.00 & 0 & 0.00 & 0 & 0 & 18 & 0 & 18 & 1 & 0 & 0 \\
\hline 31 & 35 & 241 & 2.17 & 231 & 8.38 & 10 & 0 & 27 & 0 & 27 & 1 & 0 & 0 \\
\hline 32 & 37 & 0 & 0.00 & 0 & 0.00 & 0 & 0 & 6 & 0 & 6 & 0 & 0 & 0 \\
\hline 33 & 39 & 0 & 0.00 & 0 & 0.00 & 0 & 0 & 6 & 0 & 6 & 0 & 0 & 0 \\
\hline 34 & 39 & 0 & 0,00 & 0 & 0.00 & 0 & 0 & 36 & 0 & 36 & 1 & 0 & 0 \\
\hline 35 & 39 & 0 & 0.00 & 0 & 0.00 & 0 & 0 & 8 & 0 & 8 & 0 & 0 & 0 \\
\hline 36 & 40 & 215 & 1.93 & 176 & 6.38 & 39 & 0 & 37 & 0 & 37 & I & 0 & 0 \\
\hline 37 & 41 & 0 & 0.00 & 0 & 0.00 & 0 & 0 & 47 & 0 & 47 & 2 & 0 & 0 \\
\hline 38 & 43 & D & 0.00 & 0 & 0.00 & 0 & 0 & 15 & 0 & 15 & 1 & 0 & 0 \\
\hline 39 & 45 & 103 & 0.93 & 103 & 3.73 & 0 & 0 & 2 & 0 & 2 & 0 & 0 & 0 \\
\hline 40 & 50 & 50 & 0.45 & 49 & 1.78 & 1 & 0 & 127 & 1 & 127 & 5 & 0 & 0 \\
\hline 41 & 54 & 0 & 0.00 & 0 & 0,00 & 0 & 0 & 10 & 0 & 10 & 0 & 0 & 0 \\
\hline 42 & 55 & 124 & 1.12 & 124 & 4.50 & 0 & 0 & 0 & 0 & 0 & 0 & 0 & 0 \\
\hline 43 & 65 & 10 & 0.09 & 10 & 0.36 & 0 & 0 & 0 & 0 & 0 & 0 & 0 & 0 \\
\hline 44 & 87 & 20 & 0.18 & 20 & 0.73 & 0 & 0 & 20 & 0 & 20 & 1 & 0 & 0 \\
\hline 45 & 100 & 74 & 0.67 & 74 & 268 & a & 0 & 0 & 0 & 0 & $\mathbf{0}$ & 0 & 0 \\
\hline \multicolumn{2}{|c|}{ Positions in total } & 11,121 & $\cdots$ & 2,758 & $\cdots$ & 8,363 & $\cdots$ & 11,121 & - & 2,758 & $\cdots$ & 8,363 & $\cdots$ \\
\hline $\begin{array}{l}\text { Avernge } \\
\text { (in prexce }\end{array}$ & t) & 7.64 & $\ldots$ & 19.44 & $\ldots$ & 3.75 & $\ldots$ & 5.10 & $\ldots$ & 13.78 & $\ldots$ & 2.24 & $\ldots$ \\
\hline
\end{tabular}


Table 14. Lithuania: Stock of Foreign Direct Investment by Sector, 1998-2002 1/ (In units as indicated)

\begin{tabular}{lrrrrr}
\hline & 1998 & 1999 & 2000 & 2001 & 2002 \\
\hline Total (in millions of U.S. dollars) & 1,625 & 2,063 & 2,334 & 2,665 & 3,981 \\
& & & & & \\
& & & & & \\
& & & & & \\
& 11.8 & 11.8 & 11.5 & 10.9 & 11.2 \\
Food, beverages and tobacco & 4.8 & 4.3 & 4.7 & 4.0 & 3.1 \\
Light industry & 15.8 & 15.7 & 12.6 & 10.7 & 15.0 \\
Manufacture of n.e.c. & 25.1 & 24.5 & 22.7 & 20.4 & 17.3 \\
Wholesale and retail trade, repair & 14.3 & 17.7 & 17.0 & 14.7 & 13.9 \\
Telecommunications & 9.8 & 13.7 & 16.2 & 19.9 & 20.1 \\
Financial intermediation & 18.4 & 12.3 & 15.3 & 19.4 & 19.4 \\
Other activities & & & & & \\
\hline
\end{tabular}

Sources: Bank of Lithuania; and Fund staff estimates.

1/ End of period. 
Table 15. Lithuania: Stock of Foreign Direct Investment by Source Country, 1998-2002 l/ (In units as indicated)

\begin{tabular}{|c|c|c|c|c|c|}
\hline & 1998 & 1999 & 2000 & 2001 & 2002 \\
\hline Total (in millions of U.S. dollars) & 1,625 & 2,063 & 2,334 & 2,665 & 3,981 \\
\hline- & \multicolumn{5}{|c|}{ (In percent) } \\
\hline United States & 18.7 & 13.4 & 9.8 & 8.3 & 8.7 \\
\hline Sweden & 16.9 & 17.5 & 17.3 & 16.1 & 15.3 \\
\hline Finland & 10.7 & 10.6 & 6.0 & 5.8 & 6.2 \\
\hline Germany & 8.2 & 7.7 & 7.4 & 9.2 & 9.6 \\
\hline United Kingdom & 6.8 & 7.1 & 6.7 & 6.5 & 5.4 \\
\hline Denmark & 6.6 & 9.7 & 18.3 & 18.6 & 17.2 \\
\hline Luxembourg & 4.6 & 3.6 & 3.4 & 2.5 & 1.2 \\
\hline Estonia & 4.3 & 4.1 & 6.4 & 10.0 & 11.7 \\
\hline Norway & 4.2 & 3.8 . & 4.3 & 3.7 & 2.9 \\
\hline Ireland & 2.8 & 2.8 & 1.2 & 0.7 & 0.2 \\
\hline Poland & 1.8 & 2.0 & 2.2 & 2.1 & 2.1 \\
\hline Russia & 1.7 & 1.4 & 1.0 & 1.6 & 5.2 \\
\hline Switzerland & 1.7 & 5.5 & 4.8 & 3.2 & 2.8 \\
\hline Austria & 1.3 & 0.7 & 0.7 & 0.4 & 0.3 \\
\hline France & 1.3 & 1.2 & 1.1 & 1.0 & 1.3 \\
\hline Italy & 1.0 & 0.3 & 0.2 & 0.4 & 0.6 \\
\hline \multicolumn{6}{|l|}{ Memorandum Item: } \\
\hline European Union & 61.3 & 63.2 & 64.3 & 64.1 & 59.5 \\
\hline
\end{tabular}

Source: Bank of Lithuania; Lithuanian Department of Statistics.

1/ End of period. 
Table 16. Lithuania: Foreign Direct Investment Inflows, 1998-2002 (In millions of U.S. dollars)

\begin{tabular}{lrrrrr}
\hline & 1998 & 1999 & 2000 & 2001 & 2002 \\
\hline FDI in Lithuania & & & & & \\
Equity capital & 925.5 & 486.5 & 378.9 & 445.8 & 732.0 \\
Reinvested eamings and other FDI & 772.1 & 371.7 & 181.4 & 260.6 & 505.2 \\
Outward FDI & 153.4 & 114.8 & 197.5 & 185.2 & 226.9 \\
& 4.2 & 8.6 & 3.7 & 7.1 & 17.6 \\
\hline
\end{tabular}

Source: Data provided by the Bank of Lithuania (Balance of Payments statistics). 
Table 17. Lithuania: International Investment Position (IIP), 1998-2002

\begin{tabular}{|c|c|c|c|c|c|}
\hline & 1998 & 1999 & 2000 & 2001 & 2002 \\
\hline & \multicolumn{5}{|c|}{ (In millions of US\$) } \\
\hline Assets & $2,472.2$ & $2,452.8$ & $2,677.6$ & $3,126.2$ & $4,225.2$ \\
\hline Direct investment abroad & 16.5 & 26.0 & 29.3 & 47.9 & 59.5 \\
\hline Equity capital and reinvested earnings & 5.3 & 16.1 & 17.6 & 26.4 & 36.3 \\
\hline Other capital & 11.1 & 9.9 & 11.7 & 21.4 & 23.2 \\
\hline Portfolio investment & 38.0 & 32.6 & 172.3 & 138.1 & 286.3 \\
\hline Equity securities & 2.9 & 5.9 & 6.0 & 4.9 & 10.6 \\
\hline Debt securities & 35.1 & 26.7 & 166.3 & 133.2 & 275.8 \\
\hline Other investment & 957.7 & $1,152.2$ & $1,117.3$ & $1,271.1$ & $1,459.6$ \\
\hline Trade credits (short-term) & 571.9 & 508.8 & 437.7 & 518.7 & 688.0 \\
\hline Loans & 41.8 & 69.6 & 357.4 & 447.8 & 288.8 \\
\hline Currency and deposits & 318.8 & 567.0 & 309.3 & 282.3 & 457.7 \\
\hline Other assets (short-term) & 25.2 & 6.8 & 12.9 & 22.3 & 25.2 \\
\hline Reserves Assets & $1,460.0$ & $1,242.1$ & $1,358.7$ & $1,669.2$ & $2,419.7$ \\
\hline Monetary gold & 50.9 & 47.1 & 47.1 & 51.5 & 63.8 \\
\hline Special drawing rights & 16.2 & 4.4 & 1.3 & 18.4 & 53.1 \\
\hline Reserve position in the Fund & 0.0 & 0.0 & 0.0 & 0.0 & 0.0 \\
\hline Foreign exchange & $1,392.9$ & $1,190.6$ & $1,310.2$ & $1,599.3$ & $2,302.8$ \\
\hline Liabilities & $4,968.4$ & $6,166.7$ & $6,693.5$ & $7,331.1$ & $9,372.5$ \\
\hline Direct investment in Lithuania & $1,625.3$ & $2,063.0$ & $2,334.3$ & $2,665.5$ & $3,981.3$ \\
\hline Equity capital and reinvested eamings & $1,133.7$ & $1,626.6$ & $1,809.5$ & $2,069.5$ & $3,106.7$ \\
\hline Other capital & 491.6 & 436.4 & 524.8 & 596.0 & 874.6 \\
\hline Portfolio investment & 407.9 & 878.5 & $1,140.4$ & $1,312.6$ & $1,511.3$ \\
\hline Equity securities & 106.9 & 106.9 & 128.1 & 95.6 & 110.9 \\
\hline Debt securities & 301.1 & 771.6 & $1,012.3$ & $1,217.1$ & $1,400.3$ \\
\hline Other investment & $2,935.1$ & $3,225.2$ & $3,218.9$ & $3,353.0$ & $3,869.8$ \\
\hline Trade credits (short-term) & 647.9 & 691.6 & 732.3 & 862.3 & $1,163.4$ \\
\hline Foreign loans & $2,101.6$ & $2,294.6$ & $2,261.8$ & $2,149.0$ & $2,273.4$ \\
\hline On behalf of the State & 947.1 & $1,119.2$ & 958.8 & 845.1 & 789.8 \\
\hline Banks & 302.3 & 342.2 & 412.3 & 474.0 & 652.1 \\
\hline Other sectors & 852.2 & 833.2 & 890.7 & 829.9 & 831.5 \\
\hline Currency and deposits & 144.8 & 189.2 & 147.9 & 166.1 & 219.3 \\
\hline Monetary authorities & 0.3 & 0.3 & 0.2 & 0.3 & 0.3 \\
\hline Banks & 144.5 & 189.0 & 147.7 & 165.8 & 219.0 \\
\hline Other sectors & 0.0 & 0.0 & 0.0 & 0.0 & 0.0 \\
\hline Other liabilities (short-term) & 40.8 & 49.8 & 76.9 & 175.6 & 213.7 \\
\hline Banks & 16.5 & 8.6 & 8.2 & 51.3 & 62.3 \\
\hline Other sectors & 24.3 & 41.2 & 68.7 & 72.8 & 93.0 \\
\hline
\end{tabular}

Source: Bank of Lithuania. 
Table 18. Lithuania: Indicators of External and Financial Vulnerability, 1999-2003

\begin{tabular}{|c|c|c|c|c|c|c|}
\hline & 1999 & 2000 & 2001 & 2002 & L.ateat & Date \\
\hline \multicolumn{7}{|l|}{ Finncial indicaton } \\
\hline State debt (domestic and forcign, in percent of GDP) $1 /$ & 28.3 & 28.5 & 27.2 & 26.0 & 26.6 & $\mathrm{Q} 1 / 2003$ \\
\hline Broad moncy (year-on-year change in percent) & 7.7 & 16.5 & 21.4 & 16.9 & 15.6 & Q1/2003 \\
\hline Broad mowey in percent of groen official reservea & 180.6 & 192.4 & 190.1 & 177.4 & 159.5 & Q1/2003 \\
\hline Private sector eredit (year-on-year change in percent) 2 & 13.8 & -1.2 & 21.4 & 30.4 & 30.8 & Q1/2003 \\
\hline \multicolumn{7}{|l|}{ Extemal indicators } \\
\hline Current account balance in perent of GDP & -11.2 & -6.0 & -4.8 & $\ldots-5.3$ & -3.7 & Q1/2003 \\
\hline Exports of GNFS (in millions of U.S. dollara) & 4,238 & 5,109 & 1,157 & 7,510 & 2,200 & Q1/2003 \\
\hline Exports of GNFS (year-0n-year change in percent) & -16.4 & 20.6 & 18.3 & 24.2 & 47.8 & Q1/2003 \\
\hline Imports of GNFS (year-on-year ahruge in pencent) & -15.9 & 9.3 & 14.8 & 24.0 & 35.9 & Q1/2003 \\
\hline Capital and financial account balance in pareat of GDP & 9.9 & 6.3 & 6.5 & 7.1 & 16.2 & Q1/2003 \\
\hline Gron official reserves (in millions of U.S. dollans) $3 /$ & 1,242 & 1,359 & 1,669 & 2,420 & 2,888 & Q1/20193 \\
\hline Gous official renerves/thort-term debt $4 /$ & 0.65 & 0.49 & 0.39 & 0.52 & 0.62 & Q1/2003 \\
\hline Grow official reserves/rewerve meney & 1.22 & 1.37 & 1.56 & 1.55 & 1.77 & Q1/2003 \\
\hline \multicolumn{7}{|l|}{ Gross official rwerves in monthe of imports of GNFS over the following yex } \\
\hline & 2.6 & 2.4 & 2.4 & 2.7 & 3.1 & Q1/2003 \\
\hline Totzal gron oxternal debt (in mitlions of U.S. doltarn) 5/ & 4,528 & 4,856 & 5,268 & 6,199 & 6,776 & Q1/2003 \\
\hline in pereent of GDP & 42.5 & 43.5 & 44.4 & 44.5 & 38.1 & Q1/2003 \\
\hline of whtch: Public wector debt (in million of U.S. dollani) & 2,392 & 2,364 & 2,332 & 2,429 & 2,844 & Q1/2003 \\
\hline in precent of GDP & 22.5 & 21.2 & I9.6 & 17.4 & 16.0 & Q1/2003 \\
\hline of which: Short-term extemal debt (in milliow of U.S. dollans) 4 & 1,899 & 2,786 & 4,315 & 4,636 & 4,636 & Q4/2002 \\
\hline in percent of grour international reserven & 152.9 & 205.1 & 258.5 & 191.6 & 191.6 & Q4/2002 \\
\hline in preent of GDP & 17.8 & 24.9 & 36.3 & 33.3 & 33.3 & Q42002 \\
\hline of which: excluding thort-tem liabilitiea of the comnercial banks 4 & 1,018 & 2,463 & 3793.0 & 3893.5 & 3893.5 & Q4/2002 \\
\hline Total net exteral debt (in millions of U.S. dollan) 6 & 2,818 & 2,948 & 3,166 & 3,645 & 4,093 & Q1/2003 \\
\hline is percent of GDP & 26.5 & 26.4 & 26.7 & 26.2 & 23.0 & Q1/2003 \\
\hline of which: Public nector dobt (in millioms of U.S. dollars) & 2,392 & 2,364 & 2,384 & 2,486 & 2,903 & Q1/2003 \\
\hline in percent of GDP & 225 & 21.2 & 20.1 & 17.8 & 16.3 & Q1/2003 \\
\hline Total net extemsl short-term debt (in million of U.S. dollars) $7 /$ & -206 & .59 & 278 & 547 & 649 & Q1/2003 \\
\hline in pereent of GDP & -0.7 & -0.5 & 2.3 & 3.9 & 3.7 & Q1/2003 \\
\hline Extema] interest payments in percent of exportu GNFS & 3.7 & 4.1 & 3.3 & 2.7 & 3.6 & Q1/2003 \\
\hline Extennal amortizstion pryments in peccent of exportu GNFS & 16.5 & I6.7 & 27.7 & 36.4 & 17.6 & Q1/2003 \\
\hline Public and publicly guaranteed debt service as percent of tax revenut & 11.5 & 12.7 & 10.1 & 4.1 & 4.1 & Q4/2002 \\
\hline Debt service as pereent of exponts of GNFS 8 / & 18.5 & 20.9 & 35.1 & 39.1 & 21.2 & Q4/2002 \\
\hline \multicolumn{7}{|l|}{ Real effoctive axchnnge nate (year-on-year change in percent, "+" = } \\
\hline approsintion) $9 /$ & 4.20 & 6.21 & -1.76 & 3.79 & 2.56 & $2 / 28 / 2003$ \\
\hline \multicolumn{7}{|l|}{ Financial market indicaton } \\
\hline Stock market index, end of period $10 /$ & 1,089 & 1,047 & 855 & 912 & 1,154 & $5 / 8 / 2003$ \\
\hline Forcign currency debt rating $11 /$ & BBB- & BBB- & BBB- & BBB & $\mathrm{BBB}+$ & 22003 \\
\hline \multicolumn{7}{|l|}{ Memorandum inem: } \\
\hline Naninal uxchenge rate (litui/U.S. dollar, end-of-period) & 4.0 & 4.0 & 4.0 & 3.3 & 3.2 & $\mathrm{Q} 1 / 2003$ \\
\hline Nominal exchunge rate (litai/erro, end-af-period) & 4.0 & 3.7 & 3.5 & 3.5 & 3.5 & Q1/2003 \\
\hline
\end{tabular}

Sources: Benk of Lithuania, Minitury of Finenee, Department of Stativtica, National Stock Exchange of Lithurnia, Bloogrberg. Baltic Newn Service, and Information Notice Syatem.

$1 /$ Pablic and publicly guanateed debt, exelwiting thort-term debt of SoDra and nonguaranteed debt of municipalitien.

$2 /$ December 2000 is adjusted for LTL 270 million of DMB's claims on private rector, which wero removed

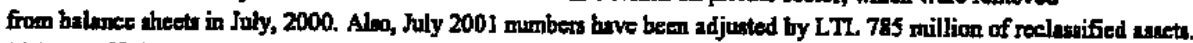

3/ Grow official resenva reported bere differ from the monetury table due to valuation differencen.

4/On a remaining meturity baris.

3/ Extemal linbilities minus equity investonent in Lithrunis.

6/ Total extemal lisbilitiou mims total extemel aueth, exchuding foreign direct investment, aquity invertment and reserve assets

$7 /$ Total short-torm litbilitiea minus total short-torn antet, on an original muturity batis.

8/ Debt service compriven intereat and repayment on extemal loans, and interest and ropayment on debt securitien.

9/ CPI bueed REER agrinat the 21 major trading parthen in 1999.

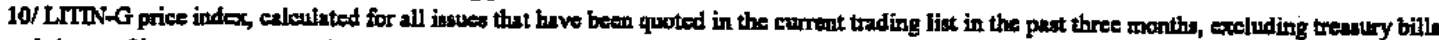
and ahares of investenent compunien.

11/ S\&P investment grado rating. 
Table 19. Lithuania: Summary of Consolidated General Government Operations, 1998-2002 1/ (In millions of Litai)

\begin{tabular}{|c|c|c|c|c|c|}
\hline & 1998 & 1999 & 2000 & 2001 & 2002 \\
\hline Total revenue & 14,036 & 13,687 & 13,707 & 14,296 & 15,112 \\
\hline Tax revenue & 13,433 & 12,955 & 12,841 & 13,154 & 13,975 \\
\hline Taxes on income and profits & 4,017 & 3,971 & 3,835 & 3,785 & 3,875 \\
\hline Taxes on companies & 579 & 361 & 312 & 259 & 308 \\
\hline Taxces on individuals & 3,438 & 3,610 & 3,523 & 3,525 & 3,568 \\
\hline Payroll tax (socjal security tax) & 3,063 & 2,891 & 3,194 & 3,265 & 3,431 \\
\hline Taxes on goods and services & 5,596 & 5,385 & 5,157 & 5,474 & 5,981 \\
\hline Of which: Excises & 1,304 & 1,643 & 1,460 & 1,643 & 1,596 \\
\hline Value-added tax & 3,612 & 3,467 & 3,419 & 3,512 & 3,810 \\
\hline Property taxes & 235 & 247 & 259 & 284 & 328 \\
\hline Foreign trade & 245 & 193 & $\mathrm{J43}$ & 134 & 132 \\
\hline Other taxes & 276 & 269 & 254 & 212 & 228 \\
\hline Nontax Revenue & 603 & 732 & 866 & 1,142 & 1,137 \\
\hline Expenditure and net lending & 16,362 & 17,152 & 15,011 & 15,065 & 15,907 \\
\hline Expenditure & 15,734 & 16,146 & 14,711 & 14,989 & 16,011 \\
\hline Current expenditure & 14,326 & 15,190 & 13,839 & 14,072 & 14,417 \\
\hline Wages and Salaries & 3,290 & 3,513 & 3,450 & 3,509 & 3,685 \\
\hline Goods and Services & 4,899 & 4,425 & 4,088 & 4,100 & 4,244 \\
\hline Transfers to households & 4,977 & 5,244 & 5,401 & 5,586 & 5,610 \\
\hline Subsidies & 202 & 150 & 103 & 81 & 126 \\
\hline Interest Payments & 507 & 650 & 782 & 781 & 687 \\
\hline Savings restitution & 450 & 1,207 & 15 & 15 & 65 \\
\hline Capital Expenditures & 1,408 & 956 & 872 & 918 & 1,594 \\
\hline Capital Transfers & 730 & 179 & 110 & 220 & 41 \\
\hline Purchases of Fixed Assets & 678 & 777 & 762 & 698 & 1,553 \\
\hline Discrepancy & 190 & 170 & -59 & 163 & 141 \\
\hline Financial balance & $-1,888$ & $-2,629$ & -944 & -856 & -707 \\
\hline Net lending & 628 & 1,006 & 300 & 76 & -104 \\
\hline Lending & 1,013 & 1,381 & 529 & 455 & 218 \\
\hline Repayments & 389 & -375 & -230 & -378 & -322 \\
\hline Fiscal balance (deficit $(-)$ ) & $-2,516$ & $-3,635$ & $-1,244$ & -932 & -603 \\
\hline Borrowing requirement & 2,516 & 3,635 & 1,244 & 932 & 603 \\
\hline Dornestic financing & -286 & 392 & -61 & 153 & 194 \\
\hline Bank financing & -666 & 337 & -109 & 105 & -215 \\
\hline Non-bank financing & 380 & 55 & 47 & 48 & 410 \\
\hline Gross Privatization receipts & 2,267 & 481 & 803 & 498 & 246 \\
\hline Foreign financing & 535 & 2,762 & 502 & 281 & 163 \\
\hline Botrowing & 1,005 & 3,441 & 1,625 & 969 & 1,635 \\
\hline Amortization & 470 & 680 & 1,123 & 689 & 1,471 \\
\hline
\end{tabular}

Sources: Ministry of Finance, Ministry of Social Security, and Fund staff estimates.

1/ From 2000 onwards, 5 new extra-budgetary funds, which had not been reported before, were added. 
Table 20. Lithuania: Summary of Consolidated General Government Operations, 1998-2002 1/ (In percent of GDP)

\begin{tabular}{|c|c|c|c|c|c|}
\hline & 1998 & 1999 & 2000 & 2001 & 2002 \\
\hline Total revenue & 32.2 & 32.1 & 30.7 & 30.1 & 29.8 \\
\hline Tax reverue & 30.8 & 30.4 & 28.7 & 27.7 & 27.6 \\
\hline Taxes on income and profits & 9.2 & 9.3 & 8.6 & 8.0 & 7.6 \\
\hline Taxes on companies & 1.3 & 0.8 & 0.7 & 0.5 & 0.6 \\
\hline Taxes on individuals & 7.9 & 8.5 & 7.9 & 7.4 & 7.0 \\
\hline Payroll tax (social security tax) & 7.0 & 6.8 & 7.1 & 6.9 & 6.8 \\
\hline Taxes on goods and services & 12.8 & 12.6 & 11.5 & 11.5 & 11.8 \\
\hline Of which: Excises & 3.9 & 3.9 & 3.3 & 3.5 & 3.1 \\
\hline Value-added tax & 8.3 & 8.1 & 7.7 & 7.4 & 7.5 \\
\hline Property tuxes & 0.5 & 0.6 & 0.6 & 0.6 & 0.6 \\
\hline Foreign trade & 0.6 & 0.5 & 0.3 & 0.3 & 0.3 \\
\hline Other taxes & 0.6 & 0.6 & 0.6 & 0.4 & 0.4 \\
\hline Nontax Revenue & 1.4 & 1.7 & 1.9 & 2.4 & 2.2 \\
\hline Expenditure and net lending & 37.6 & 40.3 & 33.6 & 31.7 & 31.4 \\
\hline Expenditure & 36.1 & 37.9 & 32.9 & 31.6 & 31.6 \\
\hline Current expenditure & 32.9 & 35.7 & 31.0 & 29.6 & 28.4 \\
\hline Wagen and Sslaries & 9.7 & 10.5 & 10.0 & 9.5 & 9.4 \\
\hline Goods and Services & 11.2 & 10.4 & 9.1 & 8.6 & 8.4 \\
\hline Transfers to househalds & 11.4 & 12.3 & 12.1 & 11.8 & 11.1 \\
\hline Subsidies & 0.5 & 0.4 & 0.2 & 0.2 & 0.2 \\
\hline Interest Payments & 1.2 & 1.5 & 1.7 & 1.6 & 1.4 \\
\hline Savinga restitution & 1.0 & 2.8 & 0.0 & 0.0 & 0.1 \\
\hline Capitil Expenditures & 3.2 & 2.2 & 2.0 & 1.9 & 3.1 \\
\hline Capital Transfers & 1.7 & 0.4 & 0.2 & 0.5 & 0.1 \\
\hline Purcheses of Fixed Assets & 1.6 & 1.8 & 1.7 & 1.5 & 3.1 \\
\hline Diserepancy & 0.4 & 0.4 & -0.1 & 0.3 & 0.3 \\
\hline Finencial balance & -4.3 & -6.2 & -2.1 & -1.8 & -1.4 \\
\hline Net Jending & 1.4 & 2.4 & 0.7 & 0.2 & -0.2 \\
\hline Lending & 2.3 & 3.2 & 1.2 & 1.0 & 0.4 \\
\hline Repayments (-) & 0.9 & -0.9 & -0.5 & -0.8 & -0.6 \\
\hline Fiscal balance (deficit $(\cdot)$ ) & -5.8 & -8.5 & -2.8 & -2.0 & -1.2 \\
\hline Borrowing requirement & 5.8 & 8.5 & 2.8 & 2.0 & 1.2 \\
\hline Dorrestic financing & -0.7 & 0.9 & -0.1 & 0.3 & 0.4 \\
\hline Bank finansing & -1.5 & 0.8 & -0.2 & 0.2 & -0.4 \\
\hline Non-bank financing & 0.9 & 0.1 & 0.1 & 0.1 & 0.8 \\
\hline Cross Privatization receipts & 5.2 & 1.1 & 1.8 & 1.0 & 0.5 \\
\hline Foreign finuncing & 1.2 & 6.5 & 1.1 & 0.6 & 0.3 \\
\hline Bocrowing & 2.3 & 8.1 & 3.6 & 2.0 & 3.2 \\
\hline Amartization & 1.1 & 1.6 & 2.5 & 1.5 & 2.9 \\
\hline \multicolumn{6}{|l|}{ Memorandum items: } \\
\hline Nominel GDP (in millions of litzi) & 43,555 & 42,608 & 44,698 & 47,498 & 50,679 \\
\hline
\end{tabular}

Sources: Ministry of Finance, Ministry of Socinl Security, and Fund staff extinates.

// From 2000 onwards, 5 new extra-budgetary funds, which had not been reported before, were added. 
Table 21. Lithuania: Social Insurance Fund (SoDra), 1998-2002

(In millions of Litai, unless otherwise indicated)

\begin{tabular}{|c|c|c|c|c|c|}
\hline & 1998 & 1999 & 2000 & 2001 & 2002 \\
\hline Revenue & 4,030 & 4,284 & 4,304 & 4,438 & 4,570 \\
\hline Mandatory social security contributions & 3,841 & 3,861 & 4,194 & 4,345 & 4,489 \\
\hline Voluntary Contributions & 0 & 0 & 1 & 1 & I \\
\hline Transfer from State Budget $1 /$ & 0 & 349 & 90 & 0 & 0 \\
\hline Other revenue & 188 & 74 & 19 & 93 & 80 \\
\hline Expenditure & 4,062 & 4,395 & 4,379 & 4,451 & 4,461 \\
\hline Pensions & 2,997 & 3,168 & 3,257 & 3,246 & 3,325 \\
\hline Allowances and other benefits & 422 & 469 & 448 & 344 & 333 \\
\hline Unemployment and accident insurance & 208 & 177 & 167 & 190 & 201 \\
\hline Transfer to Health Insurance Fund & 363 & 304 & 356 & 356 & 371 \\
\hline Cost of social insurance fund institutions & 158 & 278 & 150 & 317 & 230 \\
\hline Balance & -32 & -110 & -75 & -13 & 109 \\
\hline Bajance excluding transfers from state budget & -32 & -410 & -171 & -13 & 109 \\
\hline \multicolumn{6}{|l|}{ Memorandum items: } \\
\hline GDP & 43,555 & 42,608 & 44,698 & 47,498 & 50,679 \\
\hline Revenue (in percent of GDP) & 9.4 & 10.0 & 9.5 & 9.3 & 9.0 \\
\hline Expenditure (in percent of GDP) & 9.4 & 10.3 & 9.7 & 9.4 & 8.8 \\
\hline Balance (in percent of GDP) & -0.1 & -0.3 & -0.2 & 0.0 & 0.2 \\
\hline Balance exciuding transfers from state budget (in percent of GDP) & -0.1 & -1.0 & -0.4 & 0.0 & 0.2 \\
\hline
\end{tabular}

Sources: SoDra; and Fund staff estimates and projections.

$1 /$ Including transfers and lending from the state budget for deficit financing. 
Table 22. Lithuania: Monetary Authorities, 1998-2003

\begin{tabular}{|c|c|c|c|c|c|c|c|}
\hline & \multirow{2}{*}{$\begin{array}{l}1998 \\
\text { Dec. }\end{array}$} & \multirow{2}{*}{$\begin{array}{l}1999 \\
\text { Dec. }\end{array}$} & \multirow{2}{*}{$\begin{array}{r}2000 \\
\text { Dec. }\end{array}$} & \multirow{2}{*}{$\begin{array}{r}2001 \\
\text { Dec. }\end{array}$} & \multirow{2}{*}{$\begin{array}{c}2002 \\
\text { Dec. }\end{array}$} & \multicolumn{2}{|c|}{2003} \\
\hline & & & & & & Mar. & Muy \\
\hline & \multicolumn{7}{|c|}{ (In millions of litai) } \\
\hline Net Foreign Assets & 4,836 & 4,054 & 4,607 & 5,818 & 7,355 & 8,874 & 7,734 \\
\hline Gold & 203 & 188 & 188 & 206 & 211 & 202 & 200 \\
\hline Convertible currencies & 4,625 & 3,857 & 4,411 & 5,604 & 7,136 & 8,664 & 7,526 \\
\hline$o / w$ Use of IMF credit & 1,011 & 922 & 767 & 604 & 399 & 211 & 174 \\
\hline Other foreign assets & 8 & 8 & 8 & 8 & 8 & 8 & 8 \\
\hline Net Domestic Assets & -575 & 35 & -655 & $-1,538$ & $-2,187$ & $-3,596$ & $-2,490$ \\
\hline Net credit to Government & -947 & -326 & -804 & $-1,510$ & $-1,972$ & $-3,353$ & $-2,168$ \\
\hline Credit to banks & 52 & 30 & 24 & 15 & 16 & 14 & 14 \\
\hline Credit to private sector & 7 & 6 & 6 & 6 & 8 & $\mathbf{8}$ & 8 \\
\hline Claims on non-bank financial institutions & 7 & 20 & 0 & $\mathbf{0}$ & 0 & 0 & 0 \\
\hline Capital accounts (with opposite sign) & 112 & 90 & -117 & -270 & -453 & -448 & -532 \\
\hline Other items, net & 193 & 214 & 237 & 220 & 215 & 184 & 189 \\
\hline Reserve Money & 4,260 & 4,088 & 3,953 & 4,280 & 5.168 & 5,278 & 5,244 \\
\hline Currency outside BoL & 3,036 & 2,972 & 2,904 & 3,263 & 4,218 & 4,321 & 4,442 \\
\hline Deposit money banks' deposits & 1,212 & 1,109 & 1,037 & 1,000 & 930 & 931 & 776 \\
\hline Reserves in litai & 868 & 445 & 621 & 584 & 591 & 573 & 442 \\
\hline Reserves in foreign currency & 344 & 436 & 416 & 417 & 339 & 358 & 334 \\
\hline Time deposits & $\mathbf{0}$ & 229 & 0 & 0 & 0 & 0 & 0 \\
\hline Special deposits & $\mathbf{0}$ & $\mathbf{0}$ & 0 & $\mathbf{0}$ & 0 & 0 & 0 \\
\hline \multirow[t]{2}{*}{ Private deposits and nonmonetary financial institutions } & 13 & 7 & 12 & 17 & 20 & 26 & 27 \\
\hline & \multicolumn{7}{|c|}{ (Percentage change, year-on-year) } \\
\hline Reserve money & 28.8 & -4.0 & -3.3 & 8.3 & 20.8 & 24.6 & 29.1 \\
\hline Currency outside the Bank of Lithuania & 11.4 & -2.1 & -2.3 & 12.3 & 29.3 & 37.2 & 37.9 \\
\hline Net foreign assets & 52.5 & -16.2 & 13.7 & 26.3 & 26.4 & 46.5 & 10.4 \\
\hline Net domestic assets & -516.9 & -106.0 & -1986.7 & 134.9 & 42.1 & 97.7 & -15.5 \\
\hline Net credit to government & 208.0 & -65.6 & 146.5 & 87.7 & 30.6 & 94.5 & -24.4 \\
\hline Credit to banks & -25.6 & -42.4 & -21.3 & -35.2 & 2.9 & 0.5 & 0.6 \\
\hline
\end{tabular}

Source: Bank of Litbuania 
Table 23. Lithuania: Deposit Money Banks, 1998-2003

(In millions of Litai, end-of-period)

\begin{tabular}{|c|c|c|c|c|c|c|c|}
\hline & 1998 & 1999 & 2000 & 2001 & 2002 & \multicolumn{2}{|c|}{2003} \\
\hline & Dec. & Dec. & Dec. & Dec. & Dec. & Mar. & May \\
\hline Net foreign assets & -479 & -316 & 747 & 608 & -343 & -801 & -319 \\
\hline Reserves & 1,447 & 1,342 & 1,282 & 1,343 & 1,390 & 1,322 & 1,132 \\
\hline Currency & 236 & 232 & 245 & 342 & 460 & 391 & 356 \\
\hline Deposits at the BoL & 1,212 & 1,109 & 1,037 & 1,000 & 930 & 931 & 776 \\
\hline Required & 725 & 775 & 742 & 832 & 784 & 774 & 741 \\
\hline Excess & 486 & 334 & 294 & 168 & 146 & 157 & 34 \\
\hline Domestic Credit & 5,421 & 5,877 & 6,596 & 9,010 & 11,123 & 11,292 & 11,322 \\
\hline Claims on general government & 922 & 903 & 1,724 & 2,708 & 2,975 & 2,680 & 2,384 \\
\hline Claims on central government, net & 560 & 340 & 944 & 2,010 & 2,523 & 2,252 & 1,996 \\
\hline Treasury bills & 994 & 485 & 495 & 353 & 478 & 351 & 371 \\
\hline Government securities & 746 & 816 & 1,232 & 1,882 & 2,261 & 2,206 & 2,029 \\
\hline Loans & 123 & 171 & 104 & 161 & 103 & 77 & 76 \\
\hline Deposits (-) & 1,304 & 1,132 & 887 & 387 & 319 & 382 & 480 \\
\hline Claims on social security funds, net & -31 & 84 & 212 & 165 & -48 & -7 & 2 \\
\hline Claims on local government & 124 & 212 & 273 & 280 & 302 & 195 & 173 \\
\hline Claims on non-financial public enterprises & 270 & 267 & 294 & 253 & 198 & 240 & 212 \\
\hline Claims on private sector & 4,055 & 4,554 & 4,380 & 5,511 & 7,167 & 7,492 & 7,801 \\
\hline Of which: Businesses & 3,512 & 3,871 & 3,788 & 4,769 & 5,924 & 6,105 & 6,279 \\
\hline Households & 526 & 665 & 573 & 714 & 1,216 & 1,360 & 1,495 \\
\hline Claims on other financial institutions & 443 & 420 & 493 & 791 & 980 & 1,119 & 1,137 \\
\hline Other items, net & -872 & -678 & -843 & $-1,170$ & $-1,151$ & -985 & -989 \\
\hline Deposits & 5,518 & 6,220 & 7,782 & 9,740 & 11,020 & 10,827 & 11,146 \\
\hline Demand & 2,762 & 2,532 & 3,009 & 3,813 & 4,563 & 4,527 & 4,667 \\
\hline Time and saving & 750 & 965 & 1,218 & 1,746 & 2,844 & 2,959 & 3,056 \\
\hline Foreign currency & 2,006 & 2,723 & 3,555 & 4,181 & 3,613 & 3,341 & 3,423 \\
\hline Bonds & 0 & 5 & 0 & 51 & 10 & 11 & 12 \\
\hline
\end{tabular}

Source: Bank of Lithuania. 


$$
-27-
$$

Table 24. Lithuania: Summary Monetary Acoounts, 1998-2003 (nn millions of Litri)

\begin{tabular}{|c|c|c|c|c|c|c|c|}
\hline & \multirow{2}{*}{$\begin{array}{l}1998 \\
\text { Dee. }\end{array}$} & \multirow{2}{*}{$\begin{array}{l}1999 \\
\text { Dec. }\end{array}$} & \multirow{2}{*}{$\begin{array}{l}20000 \\
\text { Dec. }\end{array}$} & \multirow{2}{*}{$\begin{array}{l}2001 \\
\text { Dec. }\end{array}$} & \multirow{2}{*}{$\begin{array}{l}2002 \\
\text { Dec. }\end{array}$} & \multicolumn{2}{|c|}{2003} \\
\hline & & & & & & Mar. & Mary \\
\hline \multicolumn{8}{|l|}{ Moneleny Authority } \\
\hline Nel Faraign Assets & 4,836 & 4,054 & 4,607 & 5,818 & 7,355 & 8,874 & 7,734 \\
\hline Gold & 203 & 188 & 188 & 206 & 211 & 202 & 200 \\
\hline Convertible etrrencin & 4,625 & 3,857 & 4,411 & 5,604 & 7,136 & $B, 664$ & 7,526 \\
\hline otw Use of IMF credit & 1,011 & 922 & 767 & 604 & 399 & 211 & 174 \\
\hline Norsenvertibie amencies & 0 & 0 & 0 & 0 & 0 & 0 & 0 \\
\hline Ohyer fartign assets & 8 & 8 & 8 & 8 & 8 & 8 & 8 \\
\hline \multicolumn{8}{|l|}{ NDA es rasichel from calculated NEA } \\
\hline Net Dombestic Assets & -575 & 35 & -655 & $-1,538$ & $-2,187$ & $-3,596$ & $-2,490$ \\
\hline Net credit to govemment & .947 & -326 & .804 & $-1,510$ & $-1,972$ & $-3,353$ & $-2,168$ \\
\hline Credit to benks & 52 & 30 & 24 & 15 & 16 & 14 & 14 \\
\hline Credit to private sector & 7 & 6 & 6 & 6 & 8 & B & B \\
\hline Credit to son-bank finmsial instimutions & 7 & 20 & 0 & 0 & 0 & 0 & 0 \\
\hline Other iterns, net & 305 & 305 & 120 & -51 & -238 & -264 & -343 \\
\hline Reserve Montry & 4,260 & 4,088 & 3,953 & 4,280 & 5,168 & 5.278 & 5.244 \\
\hline Cunnengy culside the curtral bank & 3,036 & 2,972 & 2,904 & 3,263 & 4,278 & 4,321 & 4,442 \\
\hline Cunrency outside barks & 2,800 & 2,739 & 2,658 & 2,920 & 3,758 & 3,930 & 4,086 \\
\hline Casth in vaults of beriles & 236 & 233 & 246 & 342 & 460 & 391 & 356 \\
\hline Deposit money barles' deposits & 1,212 & 1,109 & 1,037 & 1,000 & 930 & 931 & 776 \\
\hline Reserves in litai & 868 & 445 & 621 & 584 & 591 & 573 & 442 \\
\hline Regusind renerves in foreign currency & 344 & 436 & 416 & 417 & 339 & 358 & 334 \\
\hline Time deposits & 0 & 229 & 0 & 0 & 0 & 0 & 0 \\
\hline Special deposits & 0 & 0 & 0 & $\mathbf{0}$ & 0 & 0 & 0 \\
\hline Privite and nen-monetary francial intritutions & 13 & 7 & 12 & 17 & 20 & 25 & 27 \\
\hline \multicolumn{8}{|l|}{ Benling Survey } \\
\hline Net Forrigh Aasets & 4,292 & 3,656 & 5,368 & 6,426 & 7,012 & 8,073 & 7,415 \\
\hline Monetery sutharity & 4,836 & 4,054 & 4,607 & 5,818 & 7.355 & 8,874 & 7,734 \\
\hline Benkw and other benking institurions & -544 & -398 & 761 & 608 & -343 & -801 & -319 \\
\hline Net Doweatic Axsets & 4,035 & $\$, 316$ & 5,087 & 6,265 & 7,824 & 6,758 & 7,901 \\
\hline Net elnims on goveonmant $1 /$ & -426 & 199 & 509 & 947 & 808 & -775 & 167 \\
\hline Moniotary suthority $2 /$ & .947 & -326 & $\$ 804$ & $-1,510$ & $-1,972$ & $-3,353$ & $-2,168$ \\
\hline Banks and other banking instingtions & 521 & 525 & 1,373 & 2,456 & 2,720 & 2,579 & 2,336 \\
\hline Credit to non firmeial public enterprises & 273 & 277 & 304 & 253 & 198 & 240 & 212 \\
\hline Credir to privute sector & 4,874 & 5.545 & 5,200 & 5,538 & 7,221 & 7,554 & 7,863 \\
\hline Credit to nonbenk fintucisal institutions & 470 & 468 & 513 & 791 & 980 & 1,119 & 1.337 \\
\hline Othe items, net & $-1,155$ & $-1,173$ & $\cdot 1,509$ & $-1,265$ & $-1,383$ & $-1,381$ & $-1,479$ \\
\hline Broad Money & 8,327 & 8,972 & 10,455 & 12,691 & 14,836 & 14,831 & 15,317 \\
\hline Cunfuracy outside banks & 2,800 & 2,739 & 2,658 & 2.920 & 3,758 & 3,930 & 4,086 \\
\hline Deposits & 5,527 & 6,233 & $7,79 ?$ & 9,771 & 11,078 & 10,901 & 11,231 \\
\hline In netional cturentey & 3,520 & 3,509 & 4.243 & 5,589 & 7,465 & 7,559 & 7,808 \\
\hline Sevings deposits & 750 & 972 & 1,208 & 1,765 & 2,892 & 3,018 & 3,124 \\
\hline Deminad deposits & 2,770 & 2,536 & 3,014 & 3,824 & 4,573 & 4,541 & 4,684 \\
\hline In foscigen eurrency & 2,007 & 2,725 & 3,554 & 4,181 & 3,614 & 3,341 & 3,423 \\
\hline \multicolumn{8}{|l|}{ Momorarditow itoms; } \\
\hline Reserve money (yearly percent chmpge) & 28.8 & -4.0 & -3.3 & 8.3 & 20.8 & 24.6 & 29.1 \\
\hline Broud money (yeerly percent chenge) & 14.5 & 7.7 & 16.5 & 21.4 & 16.9 & 15.6 & 18.2 \\
\hline Private bector credit (yourly pescont change) & 16.9 & 13.8 & -1.2 & 6.3 & 30.4 & 30.8 & $\mathbf{3 5 . 0}$ \\
\hline Money multipiiex & 2.0 & 2.2 & 2.6 & 3.0 & 2.9 & 2.8 & 2.9 \\
\hline Currentry / deposits, in percent & 50.7 & 43.9 & 34.1 & 29.9 & 33.9 & 36.1 & 36.4 \\
\hline Foroign currency / litai deposits, in percent & 57.0 & 77.7 & B3.8 & 74.8 & 48.4 & 44.2 & 43.8 \\
\hline Velocity of broed momey a & 5.6 & 4.9 & 4.6 & 4.1 & 3.7 & $\ldots$ & $\ldots$ \\
\hline
\end{tabular}

Sources: Bark of Lilhumita; ard Fund steff estimenes mad projections.

1/ Progrand definitions for net foreign arsets (NFA) and net intemational reaterves (NIR) of the Bank of Lithumin differ in that N5A axelude the partion of Fund drawings which is Ellocated townads impon finencing through the State Liabilities Commission (see Annex II of the MEP, EBS/9W199, for the detriled diefirition of NIR).

1/ Excludes local government depocits; includes counterpert firnde.

2/ Projectiona for 2001 onwend inctude Treasury accounts, whith were moved from commercial berios to the Bol at end-June, 2001.

3/ Deceraber 2000 is adjustod for LIL 270 million of DMA's desings on privite sector.

whibh weje jowoved from bajenee theets in July, 2000. Also, August 2001 nembers havo been adjusted by LTL. 785 million of recleasified assels. $4 /$ Baced on marige broad money. 
Table 25. Lithuania: Average Interest Rates on Treasury Bills, 1998-2003

(In percent per annum)

\begin{tabular}{|c|c|c|c|c|c|c|c|c|c|c|c|c|c|c|}
\hline & \multirow[t]{2}{*}{1998} & \multirow[t]{2}{*}{1999} & \multirow[t]{2}{*}{2000} & \multicolumn{5}{|c|}{2001} & \multicolumn{5}{|c|}{2002} & \multirow{2}{*}{$\frac{2003}{\mathrm{Q} 1}$} \\
\hline & & & & Q1 & Q2 & Q3 & Q4 & Total & Q1 & Q2 & Q3 & Q4 & Total & \\
\hline Maturity up to 31 days & 8.7 & 7.8 & $\cdots$ & $\cdots$ & $\cdots$ & $\cdots$ & $\cdots$ & $\cdots$ & $\cdots$ & $\ldots$ & $\cdots$ & $\cdots$ & $\ldots$ & \\
\hline 1 to 3 month maturity & 10.6 & 11.7 & 7.3 & $\cdots$ & $\cdots$ & $\cdots$ & $\cdots$ & $\cdots$ & $\cdots$ & $\cdots$ & $\cdots$ & $\cdots$ & $\ldots$ & .. \\
\hline 3 to 6 monith maturity & 10.6 & 10.2 & 7.5 & $\ldots$ & $\ldots$ & $\cdots$ & $\ldots$ & $\ldots$ & $\cdots$ & 3.4 & $\ldots$ & $\ldots$ & 3.4 & $\cdots$ \\
\hline 6 to 12 month maturity & 11.7 & 12.7 & 9.2 & 6.8 & 5.7 & 5.1 & 4.6 & 5.7 & 4.4 & 4.1 & 3.3 & 3.1 & 3.7 & 3.1 \\
\hline Over one year maturity & $\ldots$ & 13.7 & 11.1 & 9.0 & 7.4 & 7.0 & 6.0 & 7.2 & 5.6 & 5.4 & 5.0 & 4.8 & 5.2 & 4.9 \\
\hline
\end{tabular}

Source: Bank of Lithuania. 
Table 26. Lithuania: Interest Rates at Commercial Banks, 1998-2003 1/

(In percent per annum)

\begin{tabular}{|c|c|c|c|c|c|c|c|c|c|c|c|c|}
\hline & \multirow{2}{*}{$\begin{array}{r}1998 \\
\text { Dec. }\end{array}$} & \multirow{2}{*}{$\begin{array}{c}1999 \\
\text { Dec. }\end{array}$} & \multirow{2}{*}{$\begin{array}{r}2000 \\
\text { Dec. }\end{array}$} & \multicolumn{4}{|c|}{2001} & \multicolumn{4}{|c|}{2002} & \multirow{2}{*}{$\frac{2003}{\text { Mar. }}$} \\
\hline & & & & Mar. & Jun. & Sep. & Dec. & Mar. & Jun. & Sep. & Dec. & \\
\hline & \multicolumn{12}{|c|}{ (Transactions in domestic currency) } \\
\hline \multicolumn{13}{|l|}{ On loans } \\
\hline From 1 to 3 months & 16.7 & 14.5 & 11.5 & 9.7 & 9.5 & 9.3 & 8.2 & 7.0 & 5.6 & 5.3 & 6.3 & 7.5 \\
\hline From 3 to 6 months & 14.5 & 17.9 & 11.1 & 9.6 & 9.5 & 8.4 & 10.8 & 7.1 & 7.2 & 5.8 & 5.4 & 6.1 \\
\hline From 1 to 5 years & 10.1 & 11.2 & 10.7 & 10.9 & 10.6 & 8.8 & 8.2 & 7.1 & 7.2 & 6.7 & 6.2 & 7.1 \\
\hline \multicolumn{13}{|l|}{ On deposits } \\
\hline From 1 to 3 months & 7.1 & 8.1 & 5.7 & 5.0 & 4.5 & 4.2 & 3.7 & 3.4 & 2.5 & 2.2 & 2.1 & 2.1 \\
\hline From 3 to 6 months & 7.4 & 8.6 & 6.7 & 6.4 & 5.3 & 4.6 & 4.2 & 3.7 & 3.1 & 2.7 & 2.6 & 2.5 \\
\hline Over 2 years & 7.2 & 9.0 & 7.4 & 8.2 & 7.3 & 7.0 & 5.1 & 4.5 & 3.8 & 3.7 & 3.8 & 3.3 \\
\hline & \multicolumn{12}{|c|}{ (Transactions in foreign currencies) } \\
\hline \multicolumn{13}{|l|}{ On loans } \\
\hline From 1 to 3 months & 9.4 & 11.3 & 13.1 & 12.5 & 11.9 & 8.9 & 8.6 & 10.6 & 9.9 & 9.8 & 6.4 & 6.6 \\
\hline From 3 to 6 months & 11.3 & 11.0 & 11.3 & 10.9 & 9.8 & 10.6 & 7.2 & 7.7 & 5.4 & 6.7 & 7.4 & 5.9 \\
\hline From 1 to $S$ years & 10.7 & 11.2 & 8.9 & 9.5 & 7.2 & 7.5 & 5.7 & 5.8 & 6.0 & 5.2 & 4.2 & 5.2 \\
\hline \multicolumn{13}{|l|}{ On deposits } \\
\hline From 1 to 3 months & 4.3 & 4.4 & 4.6 & 4.4 & 3.7 & 3.4 & 2.2 & 2.0 & 1.9 & 1.8 & 1.5 & 1.6 \\
\hline From 3 to 6 months & 4.7 & 4.9 & 5.1 & 4.9 & 4.1 & 3.5 & 2.5 & 1.9 & 1.9 & 1.9 & 1.9 & 1.7 \\
\hline Over 2 years & 5.1 & 5.9 & 5.8 & 5.1 & 3.4 & 4.1 & 3.1 & 2.3 & 2.6 & 2.3 & 2.7 & 2.2 \\
\hline
\end{tabular}

Source: Bank of Lithuania.

1/ Average annual interest rates on received deposits and on loans granted in a given month. 
Table 27. Lithuania: Indicators of Financial Sector Vulnerability, 1998-2003

(In percent, unless otherwise indicated)

\begin{tabular}{|c|c|c|c|c|c|c|c|c|c|}
\hline & \multirow[t]{2}{*}{1998} & \multirow[t]{2}{*}{1999} & \multirow[t]{2}{*}{2000} & \multirow[t]{2}{*}{2001} & \multicolumn{4}{|c|}{2002} & \multirow{2}{*}{$\frac{2003}{\text { Mar. }}$} \\
\hline & & & & & Mar. & Jun. & Sept. & Dec. & \\
\hline Private sector credit (percentage change, year-on-year) $1 /$ & 16.9 & 13.8 & -1.2 & 15.4 & 21.6 & 16.1 & 33.3 & 30.4 & 30.8 \\
\hline Loans to private enterprises & 3,493 & 3,858 & 3,715 & 4,549 & 4,674 & 4,738 & 5,069 & 5,697 & 5,881 \\
\hline Laans to private enterprises (percentage change, year-on-year) & 10.3 & 10.4 & -3.7 & 22.5 & 23.3 & 22.9 & 29.1 & 25.2 & 25.8 \\
\hline Share of loans to private enterprises in total lending & 67.8 & 65.3 & 62.8 & 62.5 & 62.2 & 63.6 & 62.7 & 63.9 & 62.7 \\
\hline Commercial bank reserves (in percent of deposits) $2 /$ & 26.2 & 21.5 & 16.4 & 11.5 & 12.0 & 9.1 & 7.3 & 9.7 & 9.9 \\
\hline Cash & 4.3 & 3.7 & 3.1 & 2.9 & 2.8 & 2.4 & 2.8 & 2.9 & 2.9 \\
\hline Reserves held at the BOL & 21.9 & 17.8 & 13.3 & 8.6 & 9.3 & 6.7 & 4.6 & 6.8 & 6.9 \\
\hline Required reserves (in percent of total reserves) & 59.9 & 69.8 & 71.6 & 83.2 & 81.4 & 84.0 & 97.6 & 84.3 & 83.2 \\
\hline \multicolumn{10}{|l|}{ Official risk indicators 3 / } \\
\hline Share of non-performing loans in total loans $4 /$ & 12.9 & 12.5 & 11.3 & 8.2 & 7.7 & 7.1 & 6.6 & 6.5 & 5.3 \\
\hline Risk-weighted capital asset ratio (capital over risk-weighted assets. & 23.8 & 17.4 & 16.3 & 15.7 & 15.2 & 15.4 & 14.7 & 14.8 & 14.7 \\
\hline Leverage ratio $6 /$ & 14.9 & 11.1 & 10.2 & 9.8 & 10.3 & 11.0 & 10.8 & 10.7 & $\ldots$ \\
\hline Share of private sector credit collateralized by real estate & 12.0 & 10.3 & 9.7 & 8.8 & $\ldots$ & $\ldots$ & $\cdots$ & $\ldots$ & $\ldots$ \\
\hline \multicolumn{10}{|l|}{ Financial sector risk factors of DMB's } \\
\hline Share of foreign currency loans in total lending & 55.9 & 61.6 & 66.8 & 60.6 & 62.2 & 59.2 & 56.0 & 51.1 & 48.1 \\
\hline Share of foreign currency deposits in total deposits & 41.9 & 48.8 & 49.5 & 49.1 & 47.0 & 43.9 & 42.8 & 42.2 & 40.9 \\
\hline Short-term loans in percent of total loans & 45.9 & 41.0 & 37.9 & 31.9 & 31.6 & 29.4 & 28.5 & 27.7 & 26.5 \\
\hline Demand deposits in percent of total deposits & 62.3 & 51.4 & 48.9 & 46.5 & 46.8 & 46.1 & 48.1 & 47.2 & 47.3 \\
\hline \multicolumn{10}{|l|}{ Market assessment } \\
\hline Share price of Vilniaus Banka (in LTL, end of period) & 29 & 26 & 50 & $\ldots$ & $\ldots$ & $\ldots$ & $\ldots$ & $\ldots$ & $\ldots$ \\
\hline Spread between VILIBID and VIIIBOR $7 / 8 /$ & 268 & 296 & 233 & 194.0 & 160.0 & 161.1 & 119.8 & 134.2 & 99.2 \\
\hline Open position in foreign exchange $9 / 10 /$ & $\ldots$ & 6.0 & 3.8 & 1.9 & 10.8 & 8.2 & 6.3 & -1.5 & 10.9 \\
\hline Total loans (in millions) & 5,150 & 5,911 & 5,916 & 7,284 & 7,519 & 7,451 & 8,090 & 8,922 & 9,380 \\
\hline Total deposits (in millions) & 6,670 & 7,450 & 9,176 & 11,659 & 11,551 & 11,594 & 12,522 & 13,484 & 13,419 \\
\hline
\end{tabular}




\section{Lithuanja: Summary of the Tax System, July 2003}

\begin{tabular}{|c|c|c|c|}
\hline Tax & Tax Base & Deductions/Exemptions & Rates \\
\hline \multicolumn{4}{|c|}{ 1. Taxes on Income and Profits } \\
\hline \multirow[t]{2}{*}{ 1.1. Profit Tax } & $\begin{array}{l}\text { Imposed on profits of legal } \\
\text { persons and permanent } \\
\text { establishments. Profits are } \\
\text { revenues plus miscellaneous } \\
\text { income less costs (production } \\
\text { costs, depreciation, interest; } \\
\text { wages) }\end{array}$ & $\begin{array}{l}\text { Deductions: Charitable contributions, } \\
\text { nonprofit public organizations, etc. }\end{array}$ & $\begin{array}{l}15 \text { percent } \\
\text { Agricultural enterprises: } 0 \\
\text { Rates for enterprises employing handicapped } \\
\text { employees are reduced by } 25 \text { to } 100 \text { percent, according } \\
\text { to number of handicapped employed. } \\
\text { If before April } 1,1997 \text { a foreign investor has invested } \\
\text { foreign capital of not less than US\$2 million a three- } \\
\text { year profit tax exemption applies followed by a } 50 \\
\text { percent profit tax reduction for a further three years, } \\
\text { However the rule will expirc by te end of } 2003 \text {. }\end{array}$ \\
\hline & $\begin{array}{l}\text { Enterprises with } 10 \text { or less } \\
\text { employees and L'TL } 0.5 \text { million } \\
\text { annual tumover }\end{array}$ & & 13 percent \\
\hline \multirow[t]{3}{*}{$\begin{array}{l}\text { 1.2. Personal Income } \\
\text { Tax }\end{array}$} & $\begin{array}{l}\text { 1. Wages, salaries, bonuses of } \\
\text { residents and nonresidents } \\
\text { (withheld at source) }\end{array}$ & $\begin{array}{l}\text { Basic deduction (BD): LTL } 290 \text { plus } \\
\text { additional sum (LTL 14.5) per child } \\
\text { under } 18 \text { years old } \\
\text { Exemptions: pensions, stipends for } \\
\text { students, charitable donations etc. }\end{array}$ & Principal Job: 33 percent \\
\hline & $\begin{array}{l}\text { 2. Royalties, dividends, } \\
\text { interest, capital gains, rentals }\end{array}$ & & 15 per cent \\
\hline & 3. Business income & & $\begin{array}{l}\text { I5 per cent on gross amount or (on the choice of a } \\
\text { taxpayer) } \\
33 \text { per cent on gross amount minus eligible deductions }\end{array}$ \\
\hline 1.3 Withholding tax & 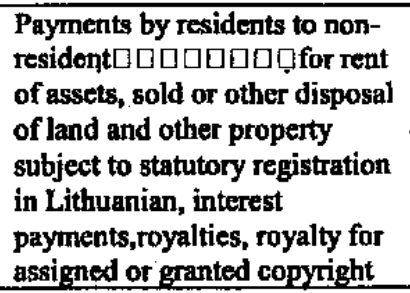 & $\begin{array}{l}\text { Exemptions: interest on government } \\
\text { bonds issued in international markets, } \\
\text { interest on deposits }\end{array}$ & 10 percent \\
\hline
\end{tabular}


Lithuania: Summary of the Tax System, July 2003

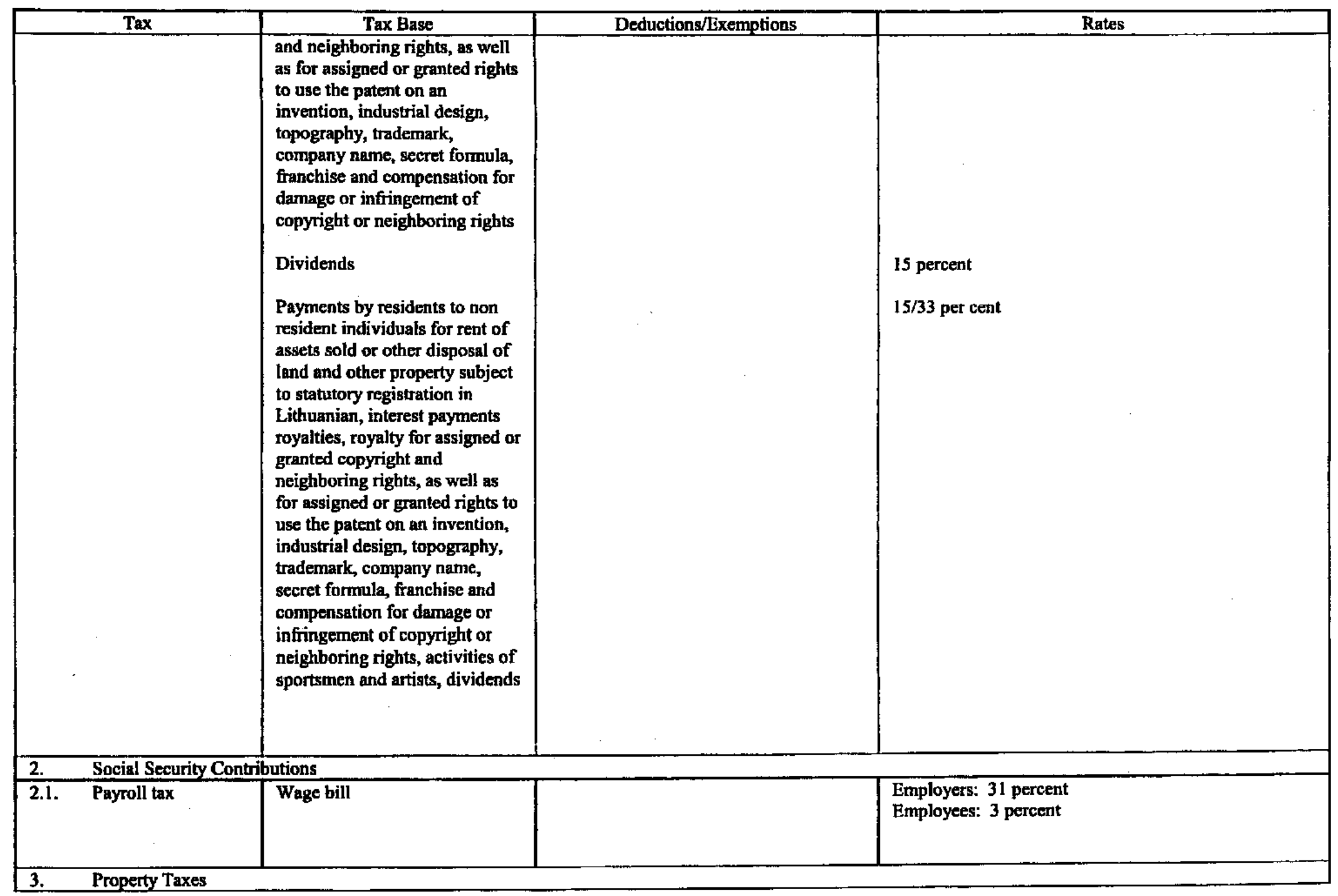


Lithuania: Summary of the Tax System, July 2003

\begin{tabular}{|c|c|c|c|}
\hline $\operatorname{Tax}$ & Tax Base & Deductions/Exemptions & Rates \\
\hline 3.1. Land tax & Value of private land & $\begin{array}{l}\text { Exemptions: } \\
\text { 1. Roads for public use. } \\
\text { 2. Land for embassies. } \\
\text { 3. Forest land etc. } \\
\text { 4. Exemptions may be granted by } \\
\text { local governments. }\end{array}$ & 1.5 percent \\
\hline & & & \\
\hline 323. Real Estate Tax & $\begin{array}{l}\text { Value of real estate owned by } \\
\text { cormpanies (non-incorporated } \\
\text { and incorporated) and other } \\
\text { organizations }\end{array}$ & $\begin{array}{l}\text { Exemptions: 1. Property owned by } \\
\text { diplomatic nissions } \\
\text { 2. Property owned by state-owned } \\
\text { enterprises. } \\
\text { 3. Property owned by societal } \\
\text { organizations. } \\
\text { 4. Cemetery and property used for } \\
\text { burial setvices. } \\
\text { 5. Property used for Activities of the } \\
\text { cult, social care and maintenance. } \\
\text { 6. Property owned by charitable } \\
\text { organizations and funds. } \\
\text { 7. Local governments may grant } \\
\text { exemptions. }\end{array}$ & 1 percent \\
\hline \multicolumn{4}{|c|}{ Tax on goods and services } \\
\hline 4.1. Value-added Tax & $\begin{array}{l}\text { Value added of goods and } \\
\text { services }\end{array}$ & $\begin{array}{l}\text { Exemptions: medicine, medical services, } \\
\text { education, culture, postal services, and } \\
\text { financial services. }\end{array}$ & $\begin{array}{l}18 \text { percent } \\
5 \text { percent for passenger transportation on approved } \\
\text { routes, newspapers, hotel accommodation, meat, certain } \\
\text { medicines } \\
6 \text { percent flat-rate scherne for small farmers } \\
0 \text { percent for exports and bio-fuels } \\
9 \text { percent for heating of housing, housing } \\
\text { construction services, financed from state funds }\end{array}$ \\
\hline 4.2. $\quad$ Excise Taxes & & & $\begin{array}{l}\text { For all excisable goods (except cigarettes from October } \\
\text { 2002) specific excises are applicable, inchuding: spirits } \\
\text { LTL 3200/hl of pure ethyl alcohol, wines of fresh } \\
\text { grapes - LTL. 150/hl, other fermented beverages having } \\
\text { an actual alcoholic strength by volume of } 8.5 \% \text { or less } \\
\text { - LTL 40/hl (other LTL 130/h), intermediate products } \\
\text { having actual alcoholic strength by volume of } 15 \% \text { or } \\
\text { less - LTL 250/hl (other LTL370/hl), beer (lll of }\end{array}$ \\
\hline
\end{tabular}


Lithuania: Summary of the Tax System, July 2003

\begin{tabular}{|c|c|c|c|c|}
\hline \multicolumn{2}{|c|}{ Tax } & \multirow{2}{*}{ Tax Base } & \multirow{2}{*}{ Deductions/Exemptions } & Rates \\
\hline & . & & & $\begin{array}{l}\text { product) - LTL } 7 / 1 \% / \text { vol/ (for } 1 \% \text { of actual alcoholic } \\
\text { strength by volume). Cigarettes excise LTL } 32 \text { per } \\
1000 \text { units (from April } 2002 \text { LTL } 36 \text { per } 1000 \text { units, } \\
\text { from October } 2002 \text { LTL } 30,2 \text { per } 1000 \text { units }+10 \\
\text { percent of the maximum retail price, from } 1 \text { March } \\
2003 \text { LTL } 42,6 \text { per } 1000 \text { units }+10 \text { per cent of } \\
\text { maximum retail selling price). Gasoline LTL } 1250 / \text { ton, } \\
\text { jet fuel, kerosene }-720 \text { LTL/ton, gas oil (diesel fuel } \\
\text { oil) - } 860 \text { LTL/ton. Liquefied gas intended for motor } \\
\text { vehicles - LTL200/10001. Heavy fuel oil LTL } 45 / \text { ton }\end{array}$ \\
\hline 4.3 & Road Tax & $\begin{array}{l}\text { Turnover tax on sales of goods } \\
\text { and services rendered. }\end{array}$ & & $\begin{array}{l}0.1-0.48 \text { percent depending on activity company is } \\
\text { engaged in (banks - } 1 \text { percent on margin) }\end{array}$ \\
\hline 5. & \multicolumn{4}{|l|}{ Other Taxes } \\
\hline 5.1. & Tax on Pollution & Pollutants & & Rates vary with quantity and type of pollutant. \\
\hline 5.2. & $\begin{array}{l}\text { Tax on Natural } \\
\text { Resources }\end{array}$ & Extraction of natural resources & & $\begin{array}{l}\text { Rates vary with nature, amount and quality of resources } \\
20 \text { percent for domestically produced oil and gas } \\
29 \text { percent if state capital is used } \\
\text { From } 1 \text { July progressive tax rate depending on amount } \\
\text { of the oil and gas extracted applies. }\end{array}$ \\
\hline 5.3 . & $\begin{array}{l}\text { Tax on Assets of } \\
\text { State-owned } \\
\text { Enterprises }\end{array}$ & $\begin{array}{l}\text { Tax represents interest paid on } \\
\text { use of state capital. Applied to } \\
\text { total assets after bank loans, } \\
\text { other debts and depreciation. }\end{array}$ & & $\begin{array}{l}\text { The basic rate is } 0.5 \text { percent, but for some activities it is } \\
0.1 \text { percent }\end{array}$ \\
\hline 5.4 . & $\begin{array}{l}\text { Fees and Charges, } \\
\text { Stamp duty }\end{array}$ & $\begin{array}{l}\text { Registration and } \\
\text { tecording of legal } \\
\text { document }\end{array}$ & $\begin{array}{l}\text { Exemptions: } \\
\text { 1. Special list of exemptions approved in } \\
\text { the Law and Government resolutions. } \\
\text { 2. Government or local govemments are } \\
\text { entitled to reduce the rate or to grant } \\
\text { exemptions when fees or charges are } \\
\text { collected into their respective budgets. }\end{array}$ & $\begin{array}{l}\text { the amount depends on expenditure of delivered } \\
\text { services which consist of: } \\
\text { 1. Cost of work for delivered services. } \\
\text { 2. Expenditure for manufacturing forms of legal } \\
\text { effect. } \\
\text { 3. Verification of execution the requirements } \\
\text { established in legal acts. }\end{array}$ \\
\hline 6. & \multicolumn{4}{|c|}{ Taxes on Foreign Trade } \\
\hline 6.1 . & Import Tariffs & Import of goods and services & & $\begin{array}{l}\text { Three-tier structure, depending on country of origin. } \\
\text { Countries with MFN status: conventional rate. } \\
\text { Countries with free trade arrangements: preferential } \\
\text { rate. All other countries: autonomous rate. } \\
\text { Rates vary widety for different product groups. } \\
\text { All tariffs are ad valorem, except for alcohol, tobacco, } \\
\text { and sugar. }\end{array}$ \\
\hline
\end{tabular}

